UCRL-ID-119970

\title{
Decision Model for Evaluating Reactor Disposition of Excess Plutonium
}

\author{
Thomas Edmunds
}

February 1995

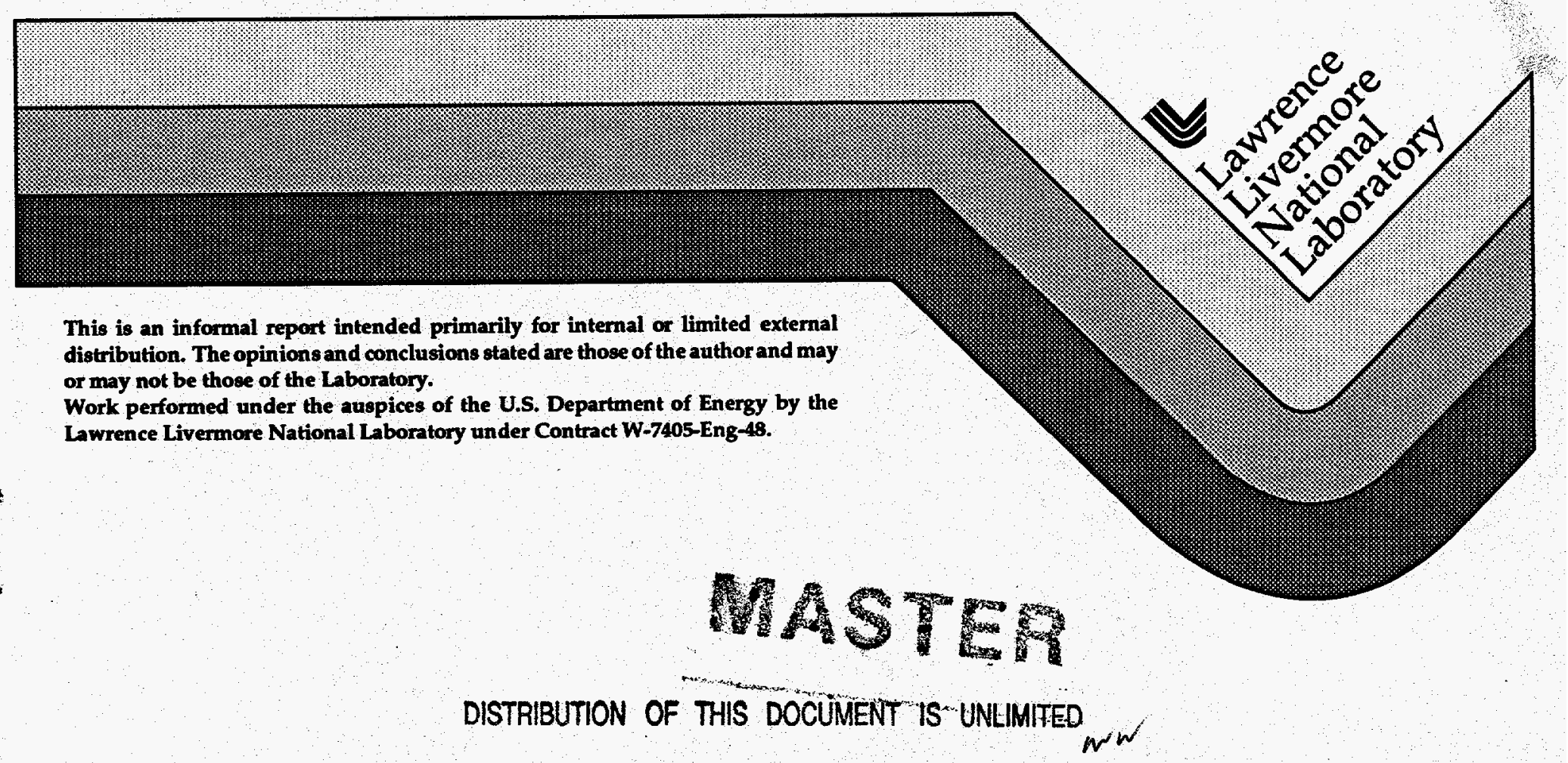




\section{DISCLAIMER}

This report was prepared as an account of work sponsored by an agency of the United States Government. Neither the United States Government nor any agency thereof, nor any of their employees, make any warranty, express or implied, or assumes any legal liability or responsibility for the accuracy, completeness, or usefulness of any information, apparatus, product, or process disclosed, or represents that its use would not infringe privately owned rights. Reference herein to any specific commercial product, process, or service by trade name, trademark, manufacturer, or otherwise does not necessarily constitute or imply its endorsement, recommendation, or favoring by the United States Government or any agency thereof. The views and opinions of authors expressed herein do not necessarily state or reflect those of the United States Government or any agency thereof. 


\section{DISCLAIMER}

Portions of this document may be illegible in electronic image products. Images are produced from the best available original document. 


\section{Decision Model for Evaluating Reactor Disposition of Excess Plutonium}

Thomas Edmunds

Lawrence Livermore National Laboratory

September 1994 


\begin{abstract}
The U. S. Department of Energy is currently considering a range of technologies for disposition of excess weapon plutonium. Use of plutonium fuel in fission reactors to generate spent fuel is one class of technology options. This report describes the inputs and results of decision analyses conducted to evaluate four evolutionary/advanced and three existing fission reactor designs for plutonium disposition. The evaluation incorporates multiple objectives or decision criteria, and accounts for uncertainty. The purpose of the study is to identify important and discriminating decision criteria, and to identify combinations of value judgments and assumptions that tend to favor one reactor design over another.
\end{abstract}




\section{Introduction}

This study is an extension of previous evaluations of options for disposition of excess plutonium ${ }^{1}$. Standard decision analysis techniques are used to support an evaluation of reactor options for plutonium disposition. Functions that measure the relative desirability of each reactor option with respect to a number of different decision criteria (e.g., life cycle cost, time to complete disposition, radiation barrier of spent fuel) are developed and combined to get a single figure of merit for each reactor. The procedures for constructing and using these functions are well developed in the multiattribute utility theory literature, have been used for DOE decision making in the past, and have been recommended by the National Academy of Sciences ${ }^{2}$. A second decision analysis technique (decision trees) is used to evaluate the impact of uncertainty regarding licensing schedules, construction schedules, and plant performance in terms of capacity factor. Standard modeling methods and a commercial computer code for implementing decision trees is used. Background material regarding decision analysis and an example application are included in Reactor Options for Disposition of Excess Weapon Plutonium: Selection Criteria and Decision Process for Assessment, LLNL Report LPDS-002, January 1994.

The decision analysis model is used to identify combinations of assumptions and value judgments that tend to favor certain reactor types. Relationships among the decision model and supporting data, assumptions and value judgments are depicted in Figure 1.

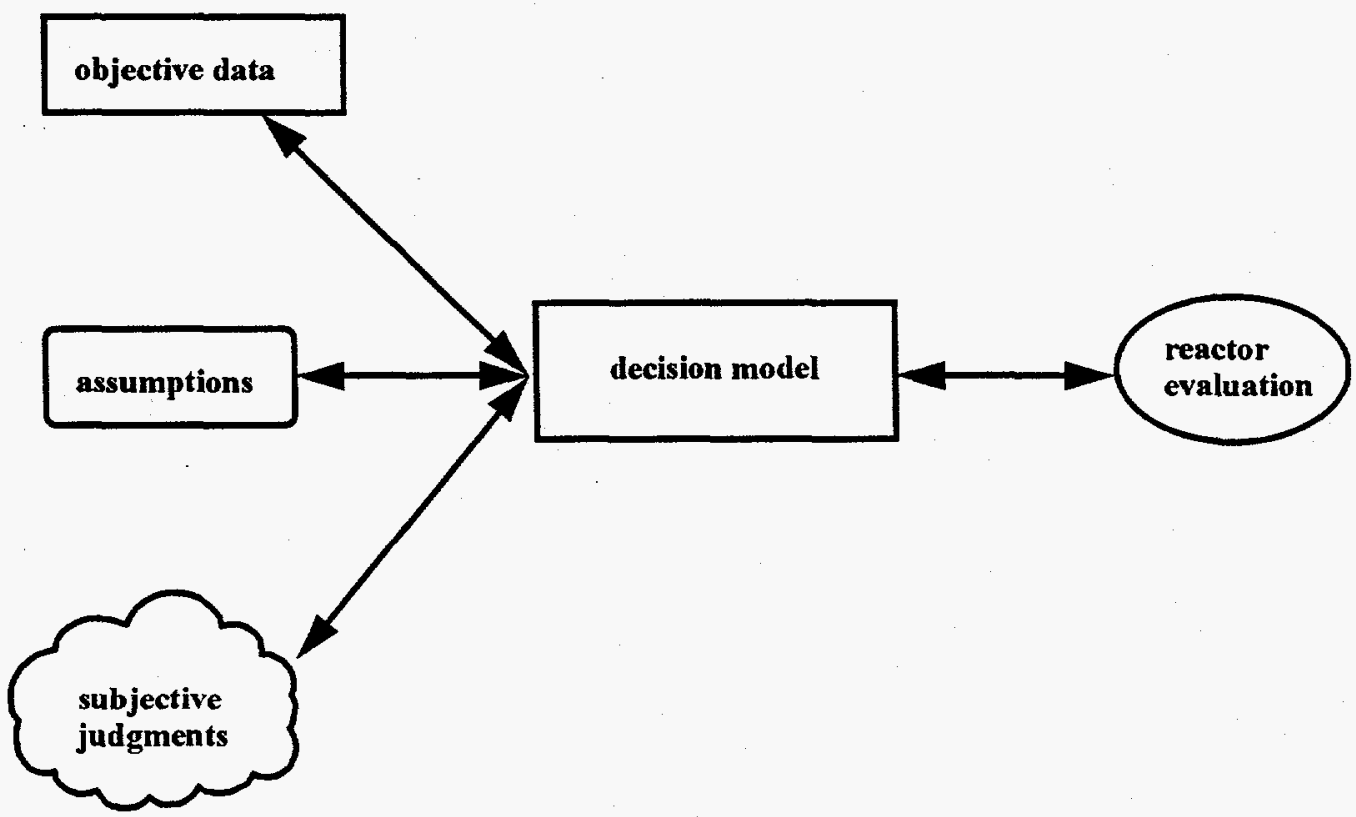

Figure 1 - Decision model inputs and outputs

Typically, decision analyses are used to support decision making, and the flow of information in Figure 1 would be from left-to-right. In this mode, data are collected, assumptions and subjective

${ }^{1}$ See U.S. DOE [1993] and Omberg and Walter [1993].

2 See DOE/RW-0074 (May 1986). This report includes a letter dated October 10, 1985 from Frank L. Parker of the National Research Council (National Academy of Sciences) to Ben C. Rusche of DOE-OCRWM. 
judgments are made, and the decision model is used to identify the optimal sequence of decisions. However, the reactor evaluation effort is intended to be a characterization of the alternatives rather than a final selection of a single reactor technology. Consequently, the flow of information for this study is from right-to-left. Specifically, a reactor is selected for evaluation and the decision model is used to identify combinations of assumptions and subjective judgments that lead to selection of that particular reactor technology.

The decision model and database are not intended to be used for final selection of a reactor technology but rather as a tool for identifying qualitative advantages and disadvantages of each reactor and to help structure a request for proposals from reactor vendors. More data should be collected before a final down select decision is made. For example, more information regarding the availability of existing reactors and the subsidies that utilities would require to switch to MOX burning operations would be needed to decide between using new or existing reactors.

\section{Decision criteria}

A list of potential decision criteria is documented in Decision Criteria for Evaluation of Plutonium Disposition Decisions, LLNL report LPDS-021, April 7, 1994. These decision criteria are intended to be comprehensive for the plutonium disposition effort, and they form the basis of an evolving decision criteria list to be used by DOE to evaluate a broader range of disposition options including, but not limited to, reactors. The evaluation criteria address a wide range of objectives, including: technical viability; proliferation resistance; environment, safety, and health; public and political acceptance; international transferability; and economics. Eighty-eight criteria are included in the comprehensive list.

Many of the criteria identified in the comprehensive list were not practical to use given the time frame and budget for the reactor evaluation effort, are not effective discriminators among the reactor options considered, or do not apply to the reactor options considered. For these reasons, a smaller set of decision criteria is derived from the comprehensive list to support development of a decision model. The criteria used in the decision model and the units used to quantify them are listed below in Table 1. 
Table 1 - Criteria used in reactor evaluation decision model

Criteria

\begin{tabular}{|r|l|l|}
\hline 1 & Life cycle cost & NPV (million 1993\$) \\
\hline 2 & Time to start disposition & years $(1993=0)$ \\
\hline 3 & Time to complete disposition & years $(1993=0)$ \\
\hline 4 & Drawdown profile & $\mathrm{MT}-\mathrm{yr}$ \\
\hline 5 & Radiation barrier at 10 years & rem/hr \\
\hline 6 & Isotopic suitability for weapons at 10 years & $\mathrm{Pu}-240 / \mathrm{Pu}$ \\
\hline 7 & Thermal suitability for weapons at 10 years & $\mathrm{kW} / \mathrm{kg} \mathrm{Pu}$ \\
\hline 8 & Net Pu destruction & fraction \\
\hline 9 & Waste generation (TRU) & cubic $\mathrm{m}$ \\
\hline 10 & Occupational dose & person-rem \\
\hline 11 & Number of sites with Pu & sites \\
\hline 12 & Radiation barrier at 100 years & rem/hr \\
\hline 13 & Initial capital outlay & $\mathrm{NPV} \mathrm{(million} 1993 \$)$ \\
\hline 14 & Number of similar reactors in Russia & reactors \\
\hline
\end{tabular}

Note that all cost and schedule calculations use 1993 as the base year. These decision criteria are used in the decision model described below.

\section{Utility functions}

\subsection{Lottery questions and single attribute utility functions}

The first step in development of the decision model is to specify a function, called a utility function, for each of the fourteen decision criteria listed above in Table 1. Each utility function reflects the desirability of achieving various values for the associated decision criterion. The utility functions for each of the fourteen decision criteria were assessed using input provided members of the Technical Evaluation Committee (TEC) assembled to evaluate the reactor options. Not all members of the TEC were interviewed. Standard utility function assessment techniques, involving lottery questions, were used (see Keeney and Raiffa 1976, and Keeney 1980).

The utility function for the first decision criterion, life cycle cost, is shown in Figure 2. The results for the other utility functions are shown in Appendix A. 


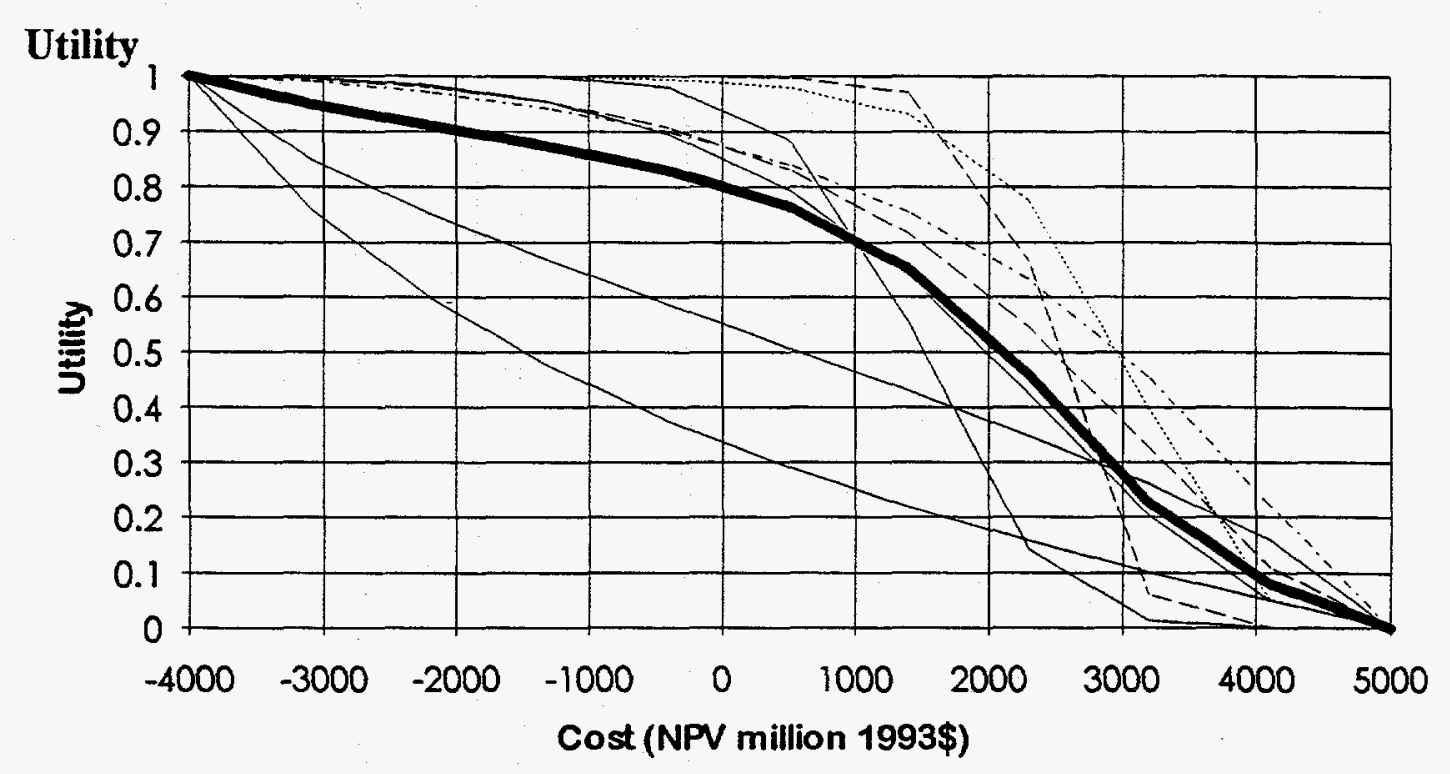

Figure 2 - Utility function for life cycle cost

The life cycle cost ranges from a low of $-\$ 4000$ million to a high of $\$ 5000$ million for utility between 1 and 0 , respectively. A negative cost implies a net profit to DOE (electricity revenues exceed costs). As shown in the figure, different TEC members specified different utility function curves. Most of the curves, however, do exhibit a general sigmoid, or "S" shape, with a transition in the range $\$-1$ to $\$+3$ billion. This reflects a general perception that DOE would view reactors that produced a profit favorably, but the level of satisfaction is not strongly dependent upon the amount of profit. The bold line in the figure is the average of the TEC member responses and is the function used in the decision model. For simplicity, the utility for a particular reactor disposition option is computed by evaluating this average function for the reactor's life cycle cost. Reactor evaluations could also be conducted for individual utility functions to determine the degree of consensus among TEC members.

\subsection{Tradeoff questions and the multiattribute utility function}

The next step in development of the model was to use responses to tradeoff questions to establish the relative importance of each of the fourteen decision criteria. In posing the tradeoff questions, TEC members were asked to construct two different options with which they were equally satisfied. The two options differed on two of the attributes. In general, one option scored well on one attribute and poorly on the other, while for the second option the scoring was reversed. An example response involving the life cycle cost and radiation barrier is shown in Table 2 .

Table 2 - Tradeoff question to define two equally preferred options

\begin{tabular}{|l|l|}
\hline \multicolumn{1}{|c|}{ Option $\mathrm{A}:$} & \multicolumn{1}{c|}{ Option B: } \\
\hline Life cycle cost $=\$ 3$ billion & Life cycle cost $=\$ 3.5$ billion \\
\hline Radiation barrier $=500 \mathrm{rem} / \mathrm{hr}$ & Radiation barrier $=1000 \mathrm{rem} / \mathrm{hr}$ \\
\hline
\end{tabular}


In this example, the TEC member is equally satisfied with options A or B. That is, the decision maker is willing to increase costs from $\$ 3$ to $\$ 3.5$ billion, or "trade off" an extra $\$ 0.5$ billion, to increase the radiation barrier performance from $500 \mathrm{rem} / \mathrm{hr}$ to $1000 \mathrm{rem} / \mathrm{hr}$ (making the spent fuel more difficult to handle and therefore more resistant to theft). The utilities for the costs and radiation barriers specified in Table 2 can be computed using the assessed utility functions. The tradeoffs between the two utility functions can then be established.

After tradeoffs between pairs of utilities have been established, a function that incorporates each of the fourteen decision criteria, called a multiattribute utility function, can be constructed. The functional form of the multiattribute utility function is a weighted sum of the fourteen single attribute utility functions, where the tradeoff questions are used to derive the weights.

$$
\mathrm{U}=\sum_{\mathrm{i}=1}^{14} \mathrm{k}_{\mathrm{i}} \mathrm{u}_{\mathrm{i}}
$$

where: $U=$ multiattribute utility function

$\mathrm{k}_{\mathrm{i}}=$ weight for single attribute utility function $\mathrm{u}_{\mathrm{i}}$

The responses to tradeoff questions posed to the TEC members were used to compute a set of weights. The results are shown in Figure 3, where the labels $k 1, \mathrm{k} 2$, etc., refer to the fourteen criteria in Table 1. The shaded bars correspond to the individual assessments and the bold bars indicate the averages of the individual assessments.

\section{Weight}

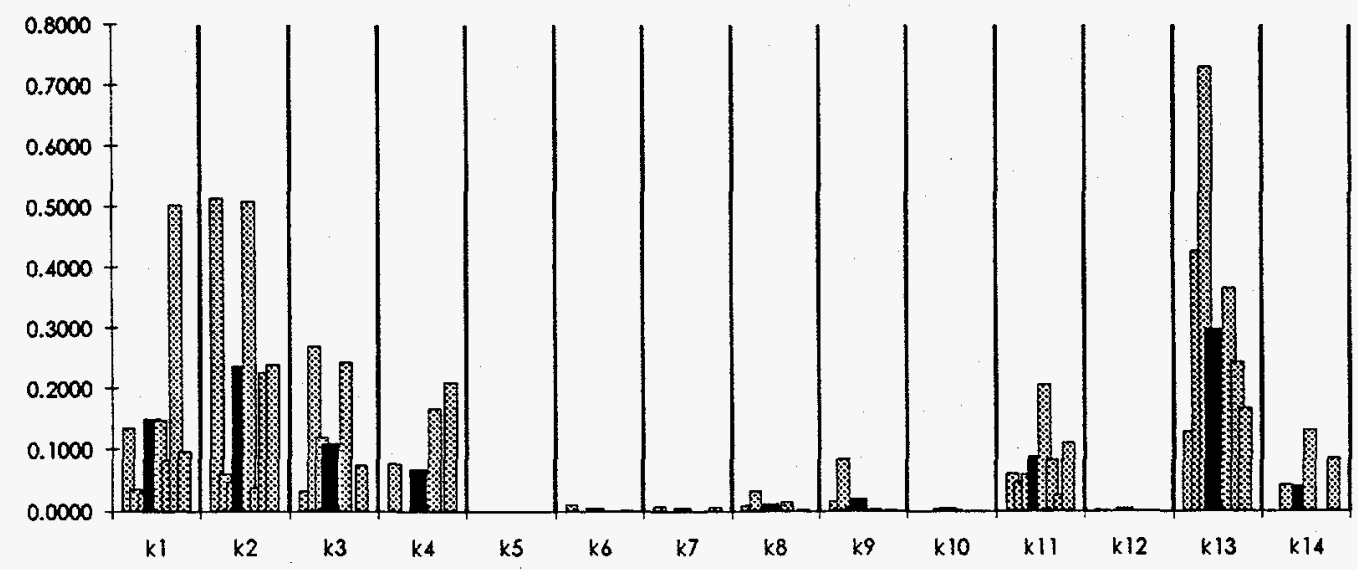

Figure 3 - Weights in multiattribute utility function (see Table 1)

As indicated by the data in the figure, life cycle cost $(\mathrm{k} 1)$, time to start $(\mathrm{k} 2)$, time to complete (k3), and initial capital cost (k13) were viewed by TEC members as most important. In general, TEC members felt that all of the proposed reactor options met the spent fuel standard ${ }^{3}$, so that the decision criteria related to spent fuel characteristics (radiation barrier, isotopic suitability for weapons, and thermal suitability for weapons) were relatively unimportant in the range of values reflected by the available reactor alternatives.

\footnotetext{
${ }^{3}$ The concept of a spent fuel standard was introduced in National Academy of Sciences [1994]. Proposed realizations of such a standard are still being developed.
} 
The utility function assessments and curve fitting routines were implemented on an electronic spreadsheet (EXCEL ${ }^{\mathrm{TM}}$ by Microsoft Corp.). Although commercial packages are available for assessment of utility functions, the spreadsheet model was used so that results could be linked to the decision tree model described in the next section. This linking capability facilitates rapid turn-around of analysis results for evaluation of the impact of changes in the utility function.

\title{
4. Decision tree
}

The spreadsheet model used to assess and fit utility functions is linked to a decision tree model implemented on a commercial decision analysis package ADA Decision Systems [1992]. The DPL code is included in Appendix B. The purpose of the decision tree model is to represent uncertainty that can impact the cost and schedule for plutonium disposition. Four major sources of uncertainty are represented in the model as probability distributions over: 1) reactor licensing delays, 2) reactor construction delays, 3) plutonium fuel fabrication facility delays, and 4) capacity factors. The following acronyms are used in the tables below.

\author{
GE - General Electric evolutionary reactor \\ W - Westinghouse advanced reactor (PDR 600) \\ $\mathrm{CE}$ - Combustion Engineering evolutionary reactor \\ GA - General Atomics advanced reactor \\ EXGE - existing General Electric reactor \\ EXW - existing Westinghouse reactor \\ EXCE - existing Combustion Engineering reactor \\ EXCAN - existing CANDU reactor
}

\subsection{Probability distribution assessments}

Four members of the TEC provided estimates of probability distributions for the evolutionary/advanced and existing reactors. Averages of these estimates are shown in Tables 3, 4,5 , and 6 . Additional analysis could be conducted using individual probability assessments to determine the degree of consensus among TEC members. As a first approximation, the probability distributions in Tables 3 through 6 are assumed to be independent of one another. 
Table 3 - Probability distribution for reactor licensing delays

\begin{tabular}{|c|c|c|c|c|c|c|}
\hline & $\begin{array}{c}-1 \text { to } 1 \\
\text { years }\end{array}$ & $\begin{array}{c}1 \text { to } 3 \\
\text { years }\end{array}$ & $\begin{array}{c}3 \text { to } 5 \\
\text { years }\end{array}$ & $\begin{array}{c}5 \text { to } 7 \\
\text { years }\end{array}$ & $\begin{array}{c}\text { not } \\
\text { licensed }\end{array}$ & sum \\
\hline GE & 0.55 & 0.23 & 0.13 & 0.08 & 0.02 & 1.00 \\
\hline W & 0.40 & 0.35 & 0.15 & 0.08 & 0.02 & 1.00 \\
\hline CE & 0.55 & 0.23 & 0.13 & 0.08 & 0.02 & 1.00 \\
\hline GA & 0.20 & 0.25 & 0.30 & 0.14 & 0.11 & 1.00 \\
\hline EXGE & 0.70 & 0.30 & 0 & 0 & 0 & 1.00 \\
\hline EXW & 0.70 & 0.30 & 0 & 0 & 0 & 1.00 \\
\hline EXCE & 0.60 & 0.40 & 0 & 0 & 0 & 1.00 \\
\hline EXCAN & 0.75 & 0.25 & 0 & 0 & 0 & 1.00 \\
\hline
\end{tabular}

Table 3 displays assessed probability distributions for delays during the licensing process for the evolutionary/advanced reactors and existing reactors, where a negative delay indicates licensing ahead of schedule. The table also includes an entry for the probability that the reactor is never licensed.

As indicated by the data in Table 3, the evolutionary/advanced reactors have a higher probability of licensing delay than the existing reactors. Among the four evolutionary/advanced reactor concepts, the GE and $\mathrm{CE}$ designs had higher probabilities of being licensed on schedule $(0.55$ probability of a delay between -1 and 1 years) than the other reactor designs. The $W$ design had slightly higher probabilities of licensing delays in the 1 to 3 and 3 to 5 year ranges, while GA design had the highest probabilities of long licensing delays. Among the existing reactors, the EXCAN design is least impacted by the introduction of MOX fuel and consequently has the lowest probability of delay. The EXW and EXGE designs introduce burnable absorbers for reactivity control.

Table 4 - Probability distribution for reactor construction delays

\begin{tabular}{|c|c|c|c|c|c|}
\hline & $\begin{array}{c}-1 \text { to } 1 \\
\text { years }\end{array}$ & $\begin{array}{c}1 \text { to } 3 \\
\text { years }\end{array}$ & $\begin{array}{c}3 \text { to } 5 \\
\text { years }\end{array}$ & $\begin{array}{c}5 \text { to } 7 \\
\text { years }\end{array}$ & sum \\
\hline GE & 0.48 & 0.28 & 0.19 & 0.06 & 1.00 \\
\hline W & 0.40 & 0.35 & 0.19 & 0.06 & 1.00 \\
\hline CE & 0.48 & 0.28 & 0.19 & 0.06 & 1.00 \\
\hline GA & 0.28 & 0.40 & 0.23 & 0.10 & 1.00 \\
\hline EXGE & 0.9 & 0.1 & 0 & 0 & 1.00 \\
\hline EXW & 0.9 & 0.1 & 0 & 0 & 1.00 \\
\hline EXCE & 0.9 & 0.1 & 0 & 0 & 1.00 \\
\hline EXCAN & 0.9 & 0.1 & 0 & 0 & 1.00 \\
\hline
\end{tabular}

The data in Table 4 indicate that of the evolutionary/advanced reactor designs, the GE and CE reactors are most likely to be constructed on schedule. The $\mathrm{W}$ design has a slightly higher probability of construction delays in the range of 1 to 3 years, while the GA design has a 
significantly higher probability of longer construction delays. The construction, or retrofit, delays for existing reactors are assumed to be low.

Table 5 - Probability distribution for fuel fabrication facility delay

\begin{tabular}{|l|c|c|c|c|c|}
\hline & $\begin{array}{c}-1 \text { to } 1 \\
\text { years }\end{array}$ & $\begin{array}{c}1 \text { to } 3 \\
\text { years }\end{array}$ & $\begin{array}{c}3 \text { to } 5 \\
\text { years }\end{array}$ & $\begin{array}{c}5 \text { to } 7 \\
\text { years }\end{array}$ & sum \\
\hline EXCAN & 0.80 & 0.20 & 0 & 0 & 1.00 \\
\hline all others & 0.50 & 0.30 & 0.10 & 0.10 & 1.00 \\
\hline
\end{tabular}

Table 5 shows the delay distributions for licensing and construction of the fuel fabrication facilities. Delay probabilities for the EXCAN facility are lower than for the other reactors because the EXCAN proposal makes use of an existing building at the DOE Hanford site (Fuels and Materials Examination Facility).

Table 6 - Probability distribution for capacity factors

\begin{tabular}{|c|c|c|c|c|c|}
\hline & $50 \%$ to $60 \%$ & $60 \%$ to $70 \%$ & $70 \%$ to $80 \%$ & $80 \%$ to $90 \%$ & sum \\
\hline GE & 0.10 & 0.28 & 0.50 & 0.12 & 1.00 \\
\hline W & 0.10 & 0.28 & 0.50 & 0.12 & 1.00 \\
\hline CE & 0.10 & 0.29 & 0.50 & 0.11 & 1.00 \\
\hline GA & 0.22 & 0.17 & 0.50 & 0.12 & 1.00 \\
\hline EXGE & 0.10 & 0.28 & 0.50 & 0.12 & 1.00 \\
\hline EXW & 0.10 & 0.28 & 0.50 & 0.12 & 1.00 \\
\hline EXCE & 0.10 & 0.29 & 0.50 & 0.11 & 1.00 \\
\hline EXCAN & 0.10 & 0.20 & 0.50 & 0.20 & 1.00 \\
\hline
\end{tabular}

The data in Table 6 indicate that there is a 0.5 probability that each reactor operates near a $75 \%$ capacity factor. The distributions for GE, $\mathrm{W}$, and $\mathrm{CE}$ evolutionary/advanced and existing reactors are almost identical, while the GA advanced reactor has a higher probability of a low (50\% to $60 \%$ ) capacity factor. The EXCAN reactor has a higher probability of achieving a high capacity factor due to the on-line refueling capability of the reactor.

\subsection{Decision tree structure}

Combinations of the chance events represented by the probability distributions in the four tables lead to a large number of possible scenarios. For example, one possible scenario is the selection of a GE reactor for licensing followed by a licensing delay in the range 1 to 3 years, a construction delay in the range 3 to 5 years, a fuel fabrication facility delay in the range 1 to 3 years, and a capacity factor in the range $50 \%$ to $60 \%$. There are 16,384 possible scenarios of this type modeled in the decision tree. Diagrams of portions of the tree are shown in Figures 4 and 5. 


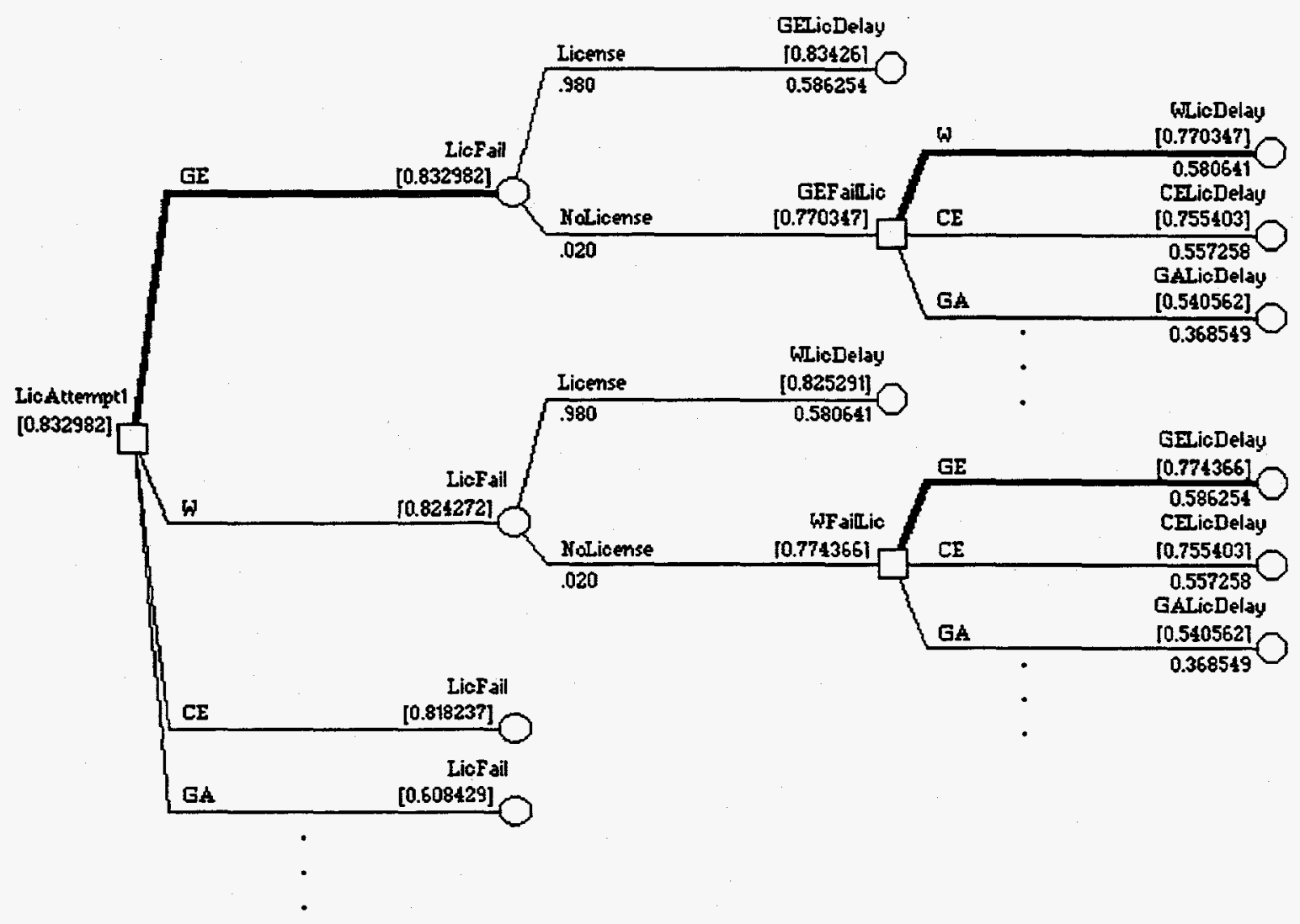

Figure 4 - Diagram of decision tree (initial decisions)

The decision tree in Figure 4 displays information for the four evolutionary/advanced reactors only. Three existing reactor designs (EXGE, EXW, and EXCAN) are included in the model but have been omitted from the figure for brevity. The modeling structure used for the existing reactors is the same as that used for the evolutionary/advanced reactors.

The squares shown in Figure 4 represent decisions and the circles represent chance nodes. The first decision, labeled "LicAttempt 1 " in the figure, is to select one of the seven reactors for licensing. The four alternatives shown in the diagram correspond to the four advanced reactor types. The figure also shows a chance event labeled "LicFail" for each of the four reactor choices shown. As indicated in the figure, the two possible outcomes of this chance event are "License" and "NoLicense." If the outcome of the chance event "LicFail" is "NoLicense," then one of the six remaining reactor designs not initially selected must be selected for a second licensing attempt. For example, if the GE reactor is selected at decision node "LicAttempt1," and the outcome of chance event "LicFail" is "NoLicense," then the decision node "GEFailLic" is encountered where the choices are W, CE, and GA reactors. As indicated in the figure, the tree structure for the W branch is similar to that for the GE branch. The CE, GA, and existing reactor branches are also similar, but they have been suppressed in the figure for brevity.

The number in brackets above each branch is the expected utility associated with the branch. The branch with the highest expected utility is selected at each decision node. The numbers beneath 
the expected utility values are the contributions for that particular branch at that particular point in the tree.

If the outcome of a "LicFail" node is "License," then a chance node representing the licensing delay, labeled "GELicDelay" in the upper branch, is encountered. The detail of the branch after the "GELicDelay" node is shown in Figure 5. Branches for the other reactor choices are similar.

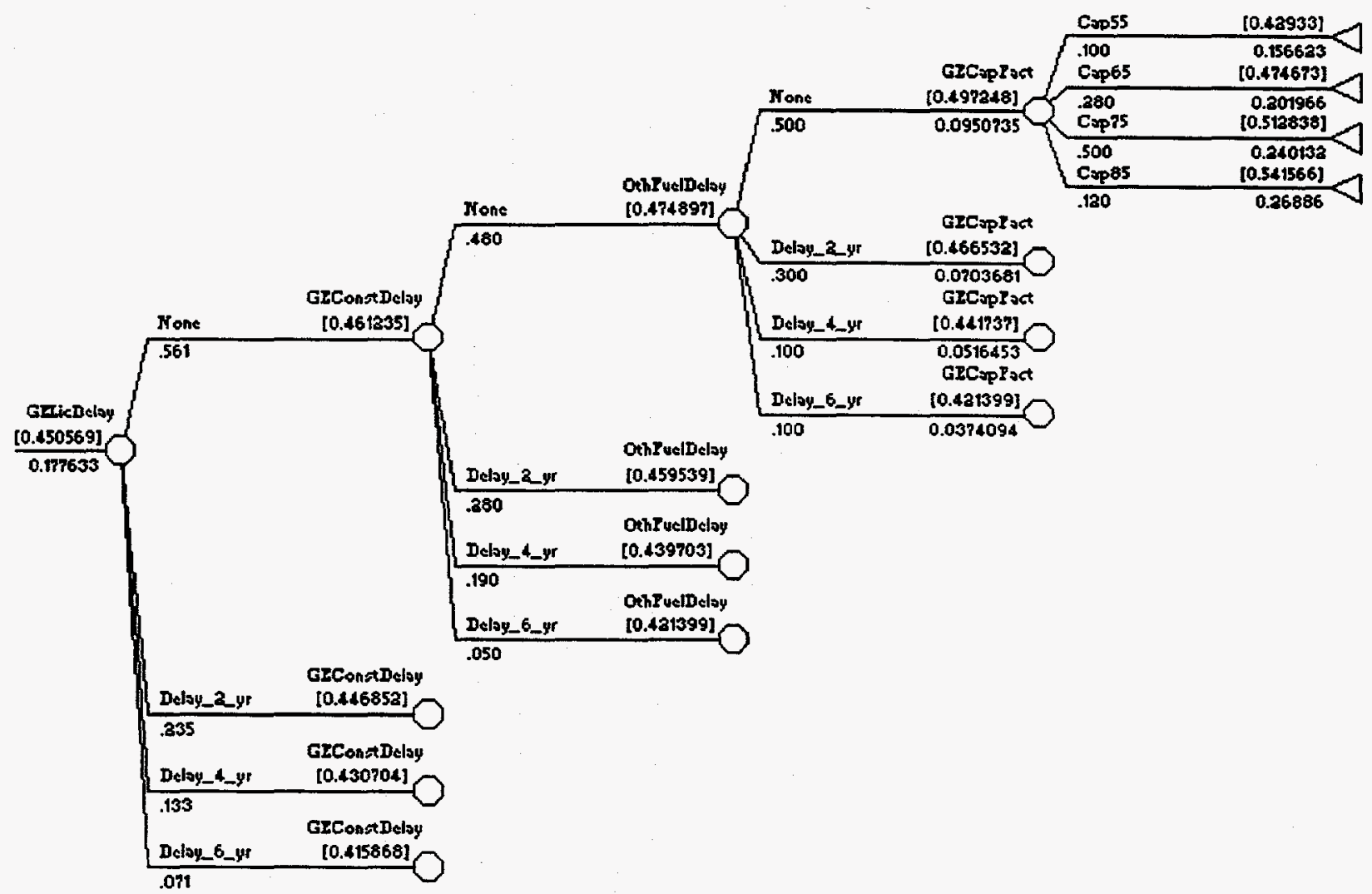

Figure 5 - Diagram of decision tree (GE branch detail)

As indicated in the figure, the possible values for licensing delays are $0,2,4$, and 6 years, which correspond to the midpoints of the intervals in the probability distribution assessment data. The next chance event encountered is labeled "GEConstDelay" in the figure. Possible values are 0, 2, 4 , and 6 years.

The next chance event labeled, "OthFuelDelay," represents delay in operations of the fuel fabrication facility. Because licensing and construction of the fuel fabrication facility is done in parallel with the reactor, the reactor and fuel plant delays are not additive. For example, if the reactor is delayed by two years and the fuel plant is delayed by 4 years, the total delay is assumed to be the maximum of the two delays, or 4 years. In the decision model, the total delay is the greater of (reactor licensing delay + reactor construction delay) and (fuel fabrication facility delay). 
Finally, the chance event for capacity factor includes $55 \%, 65 \%, 75 \%$, and $85 \%$ as possible outcomes. Full details are included for only the top branch in the figure. Other branches are similar.

\subsection{Simplified economic models}

Detailed economic models were developed for base case scenarios (no delays and nominal capacity factor of $75 \%$ ) by members of the Cost and Schedule Subcommittee of the TEC.

Simplified economic models are included in the decision tree model to estimate the impacts of delays, performance levels, electricity prices, etc. The simplified economic models incorporate three basic features.

First, each vendor's set of reactors is represented by a single "equivalent" reactor for the purposes of computing life cycle costs under different assumptions about delays, capacity factors, electricity prices, and other parameters of interest. The life cycle cost of the equivalent reactor is normalized to the values computed by the Cost and Schedule Subcommittee for the base case set of reactors for each vendor. Capital costs and revenues are computed for the equivalent reactor by modifying base case results using standard engineering economics techniques for power generation units.

Second, licensing delays are modeled as shifts in expenditures to future dates by the amount of the delay. The effect of the delay is to discount the present value of the base case expenditures by the factor $(1 /(1+d))^{n}$, where $d$ is the discount rate and $\mathrm{n}$ is the number of years licensing delay.

Third, construction delays are assumed to happen early in the construction phase. We assume that delays shift $75 \%$ of the capital costs, all of the operating costs, and all of the revenues to future dates so that they are discounted by a factor $(1 /(1+\mathrm{d}))^{\mathrm{n}}$. In addition, an annuity, with each annual payment equal to $20 \%$ of sunk capital at the time of delay, is added to construction costs during the delay period. This annuity represents the costs of retaining skilled craftsmen and engineers, of maintaining structures and the site, and of resolving the cause of the delay. Sensitivity analyses were conducted to evalute the impact of the assumed $75 \%$ sunk capital and $20 \%$ annuity rates.

\section{Results}

The decision models identify optimal reactor choices for a given set of assumptions and value judgments. In this section, we use the decision models to identify combinations of assumptions and value judgments that imply a preference of one reactor over the others. The decision models also provide a measure of the sensitivity of decisions to assumptions and value judgments. Analyses are reported for the following parameters. 
Table 6 - Parameters for sensitivity studies

\begin{tabular}{|l|c|c|c|}
\hline \multicolumn{1}{|c|}{ Parameter } & Base value & Minimum & Maximum \\
\hline start time/life cycle cost tradeoff & 1.59 & 0 & 5 \\
\hline completion time/life cycle cost tradeoff & 0.72 & 0 & 5 \\
\hline initial capital/life cycle cost tradeoff & 2.01 & 0 & 5 \\
\hline number of sites/life cycle cost tradeoff & 0.56 & 0 & 10 \\
\hline net Pu destruction/life cycle cost tradeoff & 0.60 & 0 & 5 \\
\hline electricity price real escalation rate & 0.015 & 0 & 0.03 \\
\hline
\end{tabular}

Results of these sensitivity analyses are shown in Figures 7-12.

Expected utility

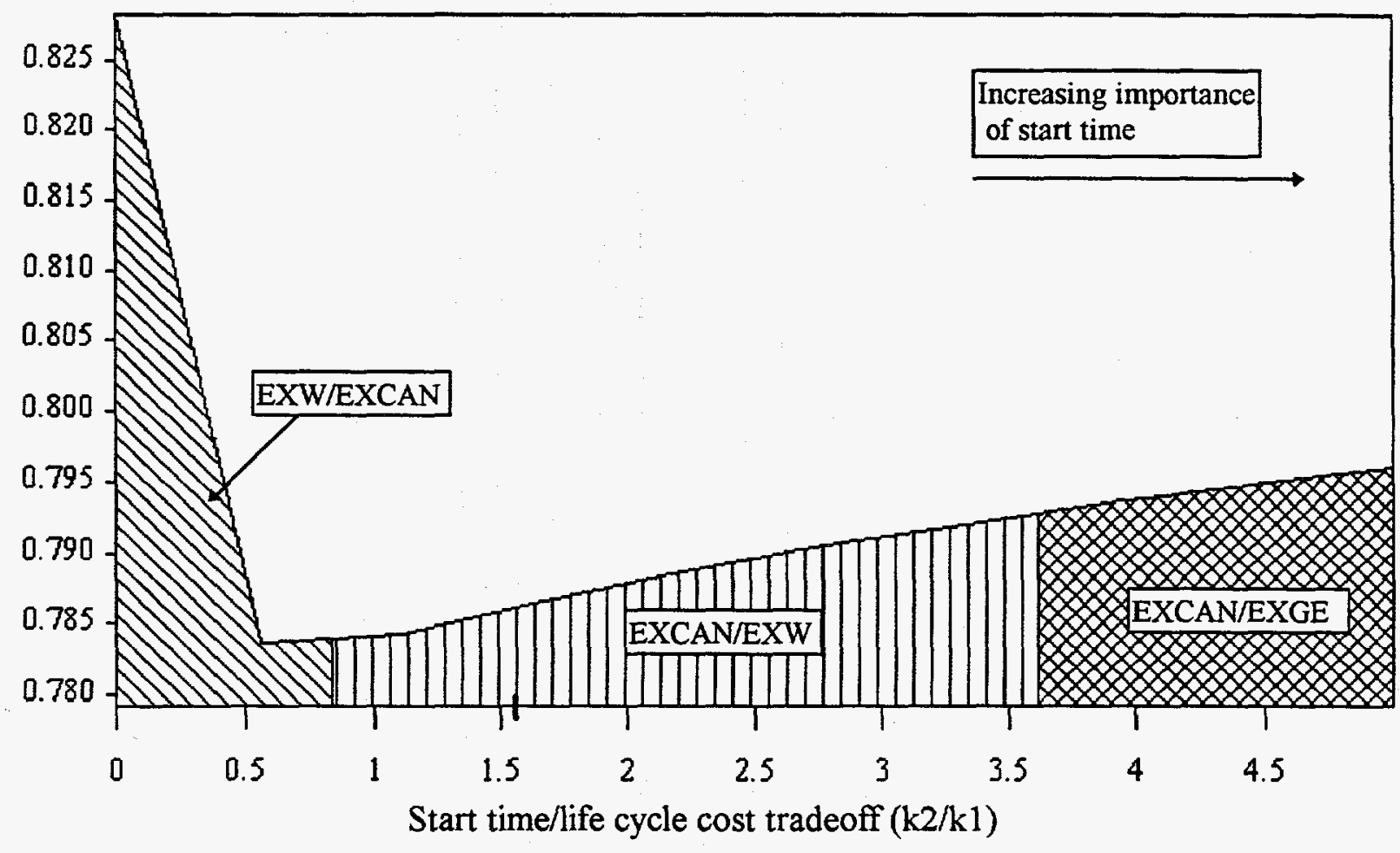

Figure 7 - Start time/life cycle cost tradeoff

Figure 7 displays the sensitivity of the outcome, as measured in expected utility, to the tradeoff between start time and life cycle cost. This tradeoff is measured as the ratio of the coefficient for start time to the coefficient for life cycle cost in the multiattribute utility function discussed in Section 3.2. A value of zero for this ratio indicates that start time in not important relative to life cycle cost, and a large value (e.g., 5.0) indicates that start time is much more important than life cycle cost. 
The labeling scheme used in Figure 7 references the reactors selected for the first and second licensing attempts depicted in Figure 4. For example, the label "EXW/EXCAN" in the figure indicates that the optimal decision sequence in the region is to initially attempt to license the existing Westinghouse (EXW) reactors (decision "LicAttempt l" in Figure 4), and to license the existing CANDU (EXCAN) reactors if the Westinghouse license attempt fails (decision "WFailLic" in Figure 4). Alternatively, the label "EXCAN/EXW" indicates that the EXCAN reactor license should be attempted first and the EXW reactor should be licensed if the EXCAN licensing attempt fails. The bold tic mark on the horizontal axis shows the tradeoff ratio assessed from members of the TEC. The overall qualitative conclusion one can draw from the figure is that the EXW reactor should be selected if schedule is unimportant relative to life cycle cost and the EXCAN reactor should be selected if the schedule is very important relative to life cycle cost.

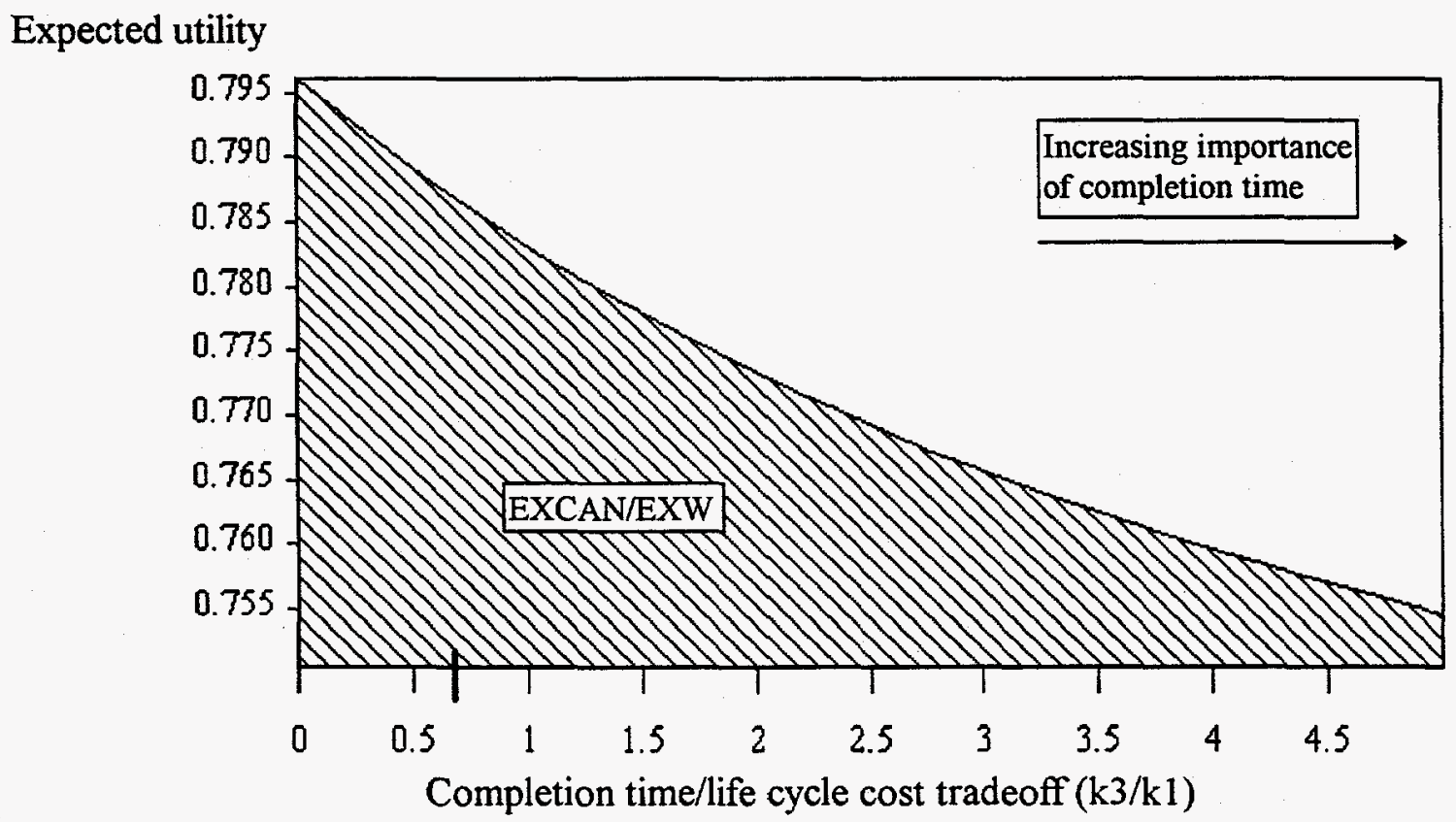

Figure 8 - Completion time/life cycle cost tradeoff

The data in Figure 8 indicate that the initial choice of the EXCAN reactor is insensitive to the completion time/life cycle cost tradeoff ratio.

A sensitivity analysis for the initial capital/life cycle cost tradeoff ratio was conducted. The ratio $\mathrm{k} 13 / \mathrm{k} 1$ was varied from a minimum of 0 (i.e., initial capital is not important) to a maximum of 5 . The initial choice of the EXCAN reactor is not sensitive to the initial capital/life cycle cost tradeoff ratio in this range.

Next, the sensitivity of the EXCAN choice to the number of sites/life cycle cost tradeoff ratio was analyzed. The ratio $\mathrm{k} 11 / \mathrm{kl}$ was varied in the range $[0,10]$. The results are shown in Figure 9. 
Expected utility

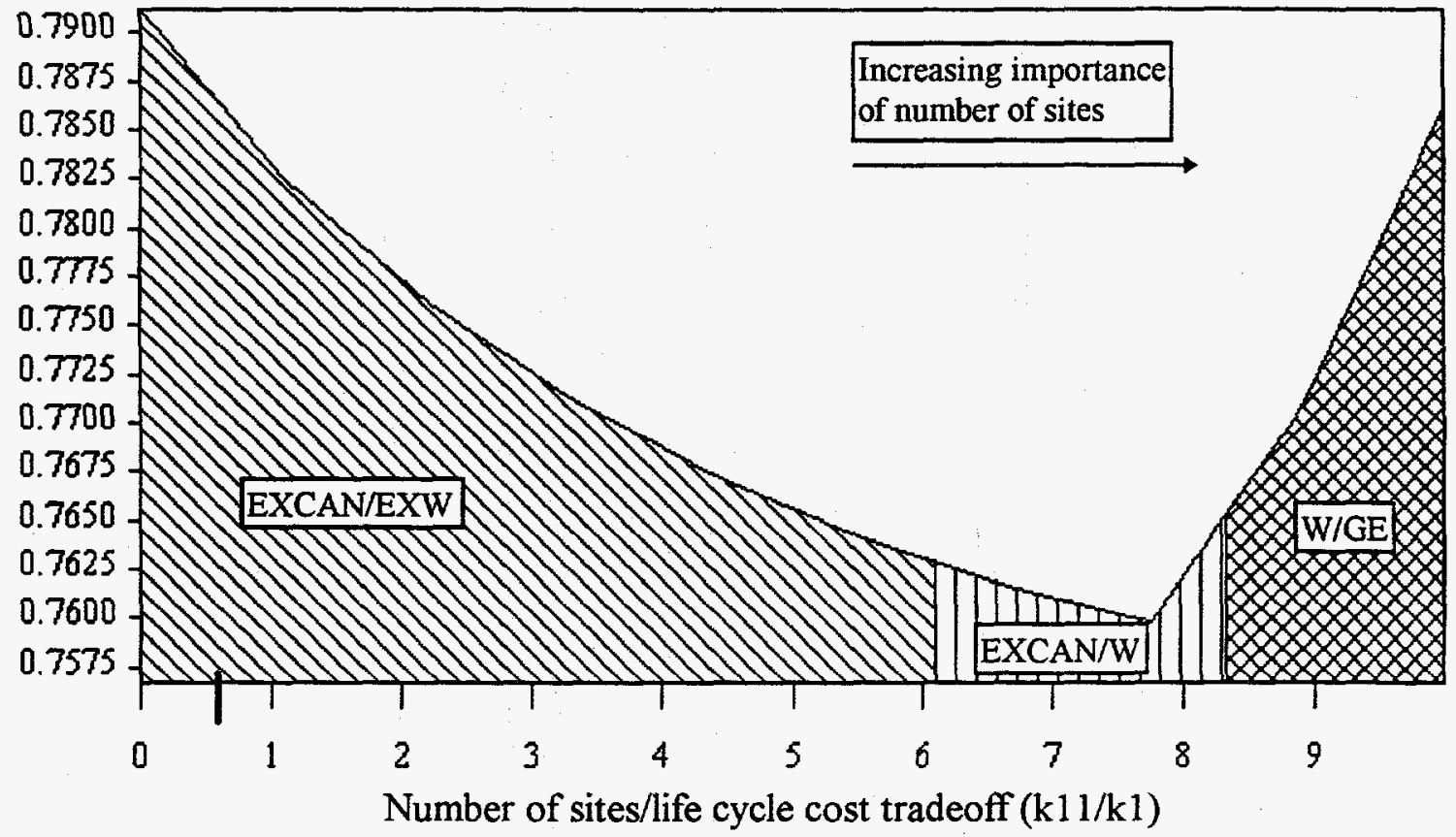

Figure 9 - Number of sites/life cycle cost tradeoff

As indicated in the figure, the CANDU reactor, which requires 2 sites, is selected if the number of sites is relatively unimportant. The advanced Westinghouse reactor, which requires a single site, is selected if the number of sites is very important.

Expected utility

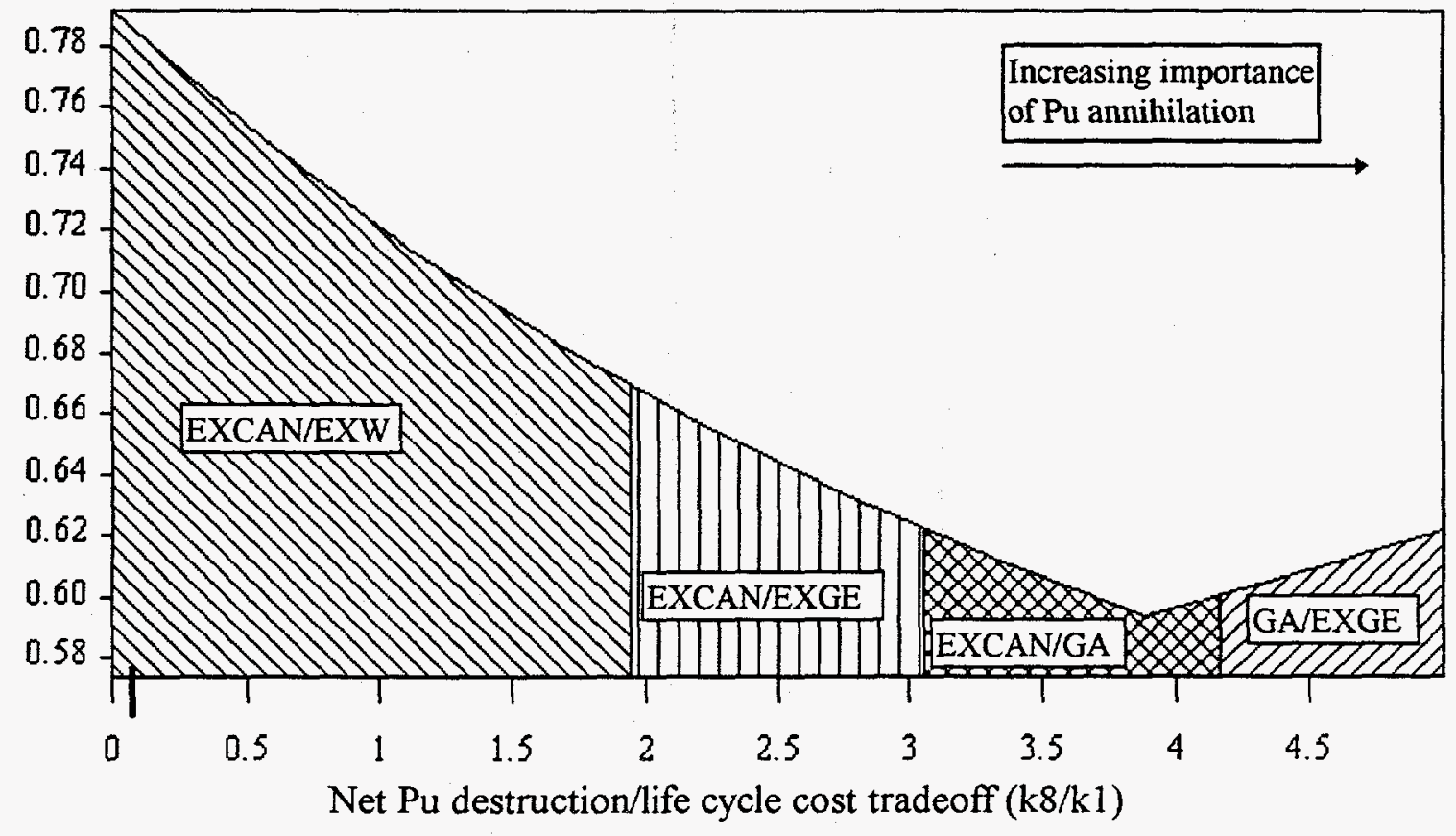

Figure 10 - Net Pu destruction/life cycle cost tradeoff 
The data shown in Figure 10 indicate that the GA reactor becomes the preferred option if net plutonium destruction is deemed to be very important. Also note that this tradeoff produces the most significant variation in expected utility. The three analyses depicted in Figures 7, 8, and 9 generate a variation in expected utility of approximately 0.04 , while the analysis depicted in Figure 10 generates a variation in expected utility of 0.2 .

Additional sensitivity analyses indicate that the value of choosing the EXCAN reactor, as measured by expected utility, is not affected by the electricity price escalation rate. This is because under the existing reactor operation scenarios all of the revenues from electricity sales belong to the utility.

However, DOE does receive the revenues from electricity sales in the advanced reactor operation scenarios. It was thought that if life cycle costs were very important and electricity price escalation rates were high then the advanced reactors might be chosen because they have high initial capital costs yet low life cycle costs when electricity prices rapidly escalate. To examine this issue further, the ratio $\mathrm{k} 13 / \mathrm{kl}$ was set to zero so that life cycle cost $(\mathrm{k} 1)$ would be considered much more important than initial capital cost (k13). The electricity price escalation sensitivity run was then repeated. The results are that existing reactors (CANDU) are preferred in this case as shown in Figure 11. Note that the expected utility changes vary little in the figure (a variation of only 0.001 ).

Expected utility

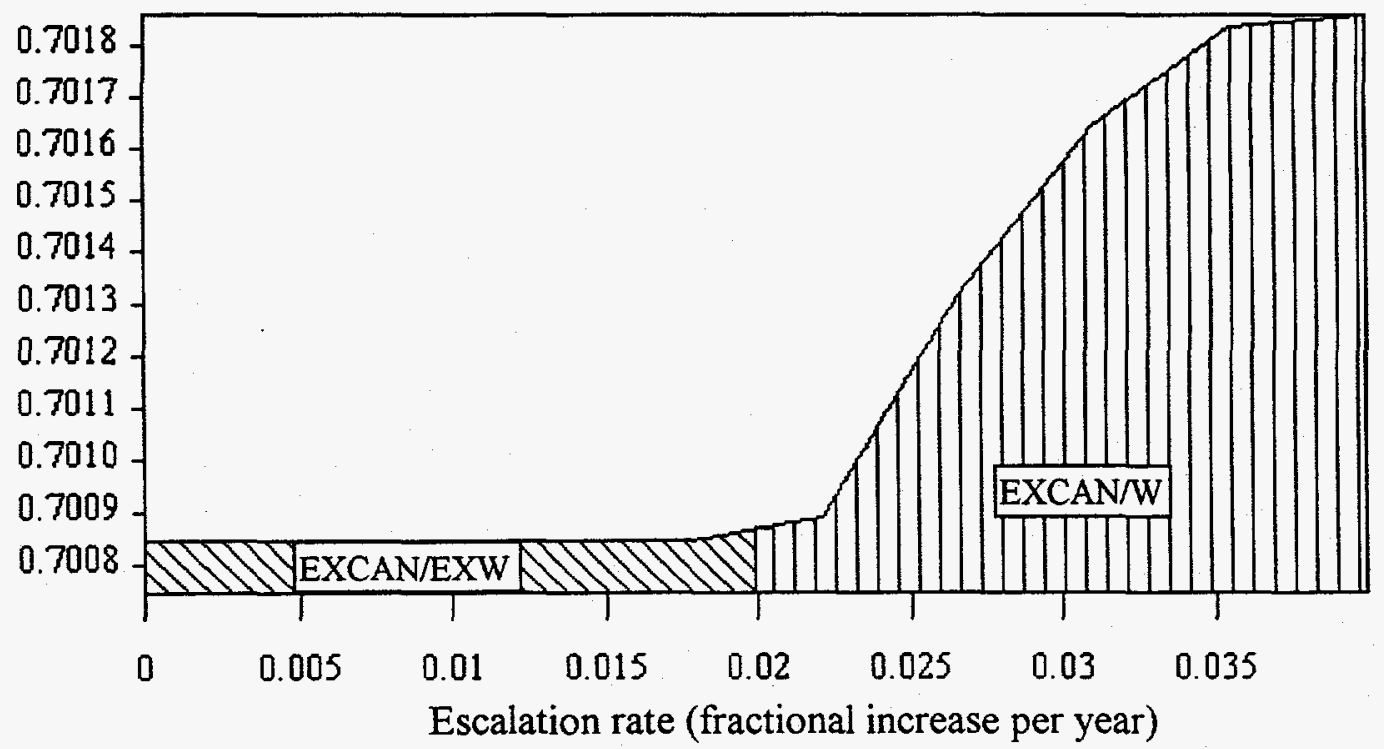

Figure 11 - Electricity price escalation rate

Finally, the coefficient for life cycle cost $(\mathrm{k} 1)$ was set equal to 1.0 and all other coefficients were set to zero. This implies that life cycle cost is the only criterion of interest. The results are shown in Figure 12. 


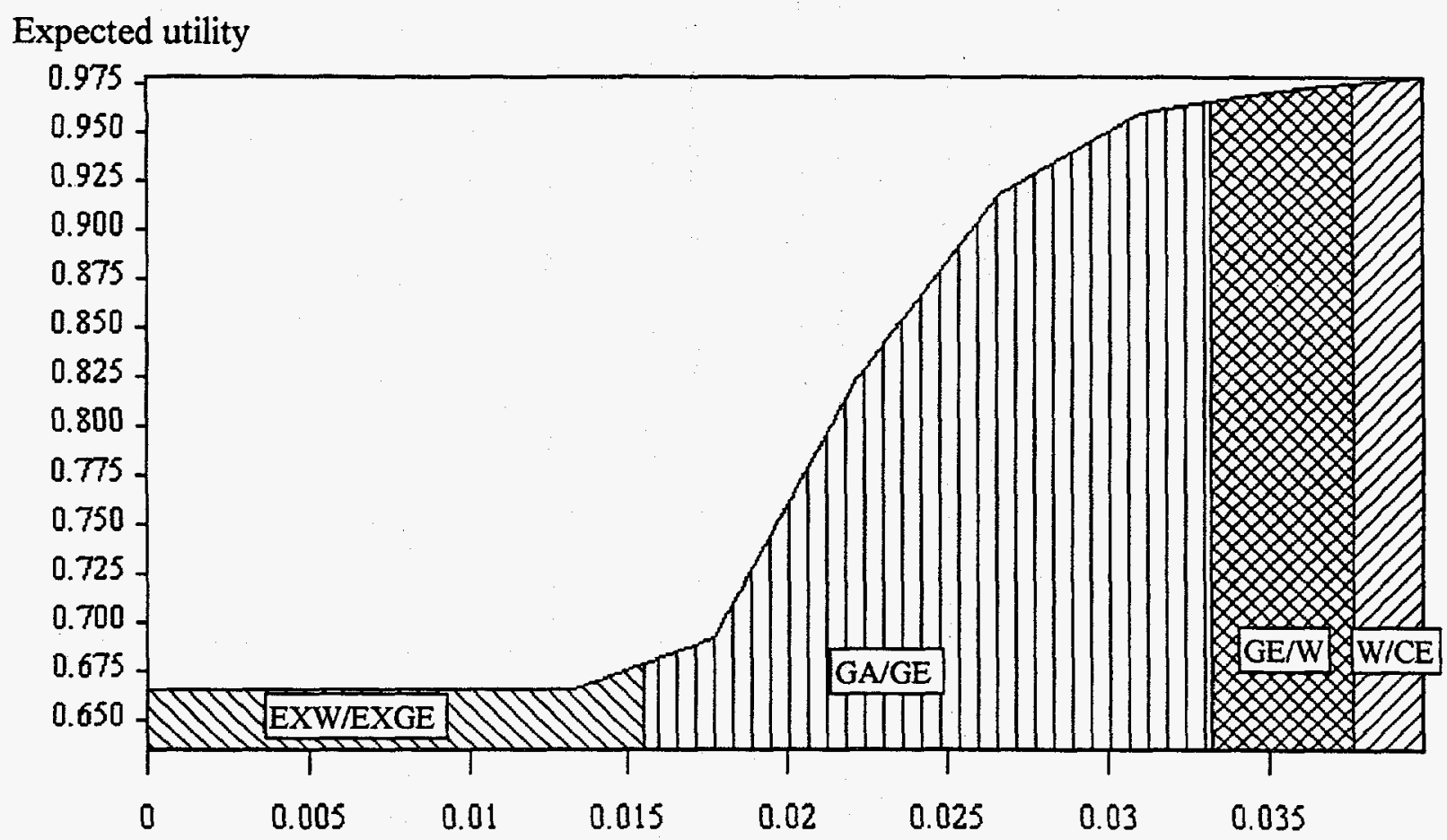

Electricity escalation rate (fractional change per year)

Figure 12 - Electricity price escalation (life cycle cost only criterion)

As indicated in Figure 12, the existing $\mathrm{W}$ reactor is preferred under low electricity escalation rates. The curve in this region is flat because revenues from electricity sales are not received by DOE. The preference switches to the GA reactor when the electricity escalation rate exceeds 0.018 (or $1.8 \%$ per year). The evolutionary GE reactor is preferred if the escalation rate is $3.5 \%$ per year, and the $\mathrm{W}$ reactor is preferred if the escalation rate is closer to $4 \%$ per year. Note the large range $(0.3)$ in expected utility for this analysis.

Results of the sensitivity analyses are summarized below.

Table 7 - Preferred reactors

\begin{tabular}{|l|c|c|c|}
\hline \multicolumn{1}{|c|}{ Parameter } & Low & Moderate & High \\
\hline importance of start time & EXW & EXCAN & EXCAN \\
\hline importance of completion time & EXCAN & EXCAN & EXCAN \\
\hline importance of initial capital & EXCAN & EXCAN & EXCAN \\
\hline importance of number of sites & EXCAN & EXCAN & W \\
\hline importance of net Pu destruction & EXCAN & EXCAN & GA \\
\hline electricity price real escalation rate & EXCAN & EXCAN & EXCAN \\
\hline electricity price escalationllife cycle cost only & GA & GE & W \\
\hline
\end{tabular}




\section{Summary and Conclusions}

In general, the existing reactor options are superior to the evolutionary/advanced reactor options with respect to two important decision criteria: initial capital cost and schedule. It requires less capital to retrofit an existing reactor to burn MOX fuel than to build a new reactor, and the time required to convert existing reactors to burn MOX fuel is less that the time required to build new reactors using plutonium based fuel. One advantage offered by the evolutionary/advanced reactors is the potential for high revenues from electricity sales that can be used by DOE to offset costs. If life cycle cost is the only criterion of interest and electricity prices increase at a rapid rate then the advanced reactor options would be preferred. One of the advanced reactors, the GA design, offers high net $\mathrm{Pu}$ annihilation, and is preferred if this criterion is given a high weight.

Among the existing reactors, the Westinghouse reactor is preferred if the time to start is unimportant relative to life cycle cost. The CANDU design is preferred under a wide range of circumstances. The existing Combustion Engineering design was not included in the evaluation due to insufficient data.

Decision criteria that were considered to be most important by some members of the TEC are: initial capital outlay, time to start, life cycle cost, time to complete, and number of sites. 


\section{References}

DPL Advanced Version User Guide, ADA Decision Systems, Menlo Park, CA [1992].

Committee on International Security and Arms Control, National Academy of Sciences, Management and Disposition of Excess Weapons Plutonium, National Academy Press, Washington, D.C. [1994].

Edmunds, Thomas, William Sutcliffe, Booth Myers, and Lisa Buonpane, "Decision Criteria for Evaluation of Plutonium Disposition Decisions", Lawrence Livermore National Laboratory, LPDS-021, [April 7, 1994].

Edmunds, Thomas, et. al., "Reactor Options for Disposition of Excess Weapon Plutonium: Selection Criteria and Decision Process for Assessment", Lawrence Livermore National Laboratory, LPDS-002 [January 1994].

Keeney, Ralph L., Siting Energy Facilities, Academic Press, New York, N.Y. [1980].

Keeney, R. L. and H. Raiffa, Decisions with Multiple Objectives, Prentice-Hall, Englewood Cliffs, N.J. [1979].

Omberg, Ronald P. and Carl E. Walter, "Disposition of Plutonium from Dismantled Weapons: Fission Options and Comparisons," UCRL-ID-113055 [February 5, 1993].

U.S. Department of Energy, Office of Civilian Radioactive Waste Management, "A Multiattribute Utility Analysis of Sites Nominated for Characterization for the First Radioactive-Waste Repository -A Decision-Aiding Methodology", DOE/RW-0074 [May 1986].

U.S. Department of Energy, Plutonium Disposition Study, "Technical Review Committee Reprot, Vol. II" [July 2, 1993]. 


\section{Appendix A - Single Attribute Utility Function Assessments}

Single attribute utility functions were assessed using standard techniques based upon lottery questions (see Keeney and Raiffa 1976). Responses to the lottery questions provide several points on the utility function curve, and a nonlinear function is fitted to the points. The assessed utility functions are shown below.

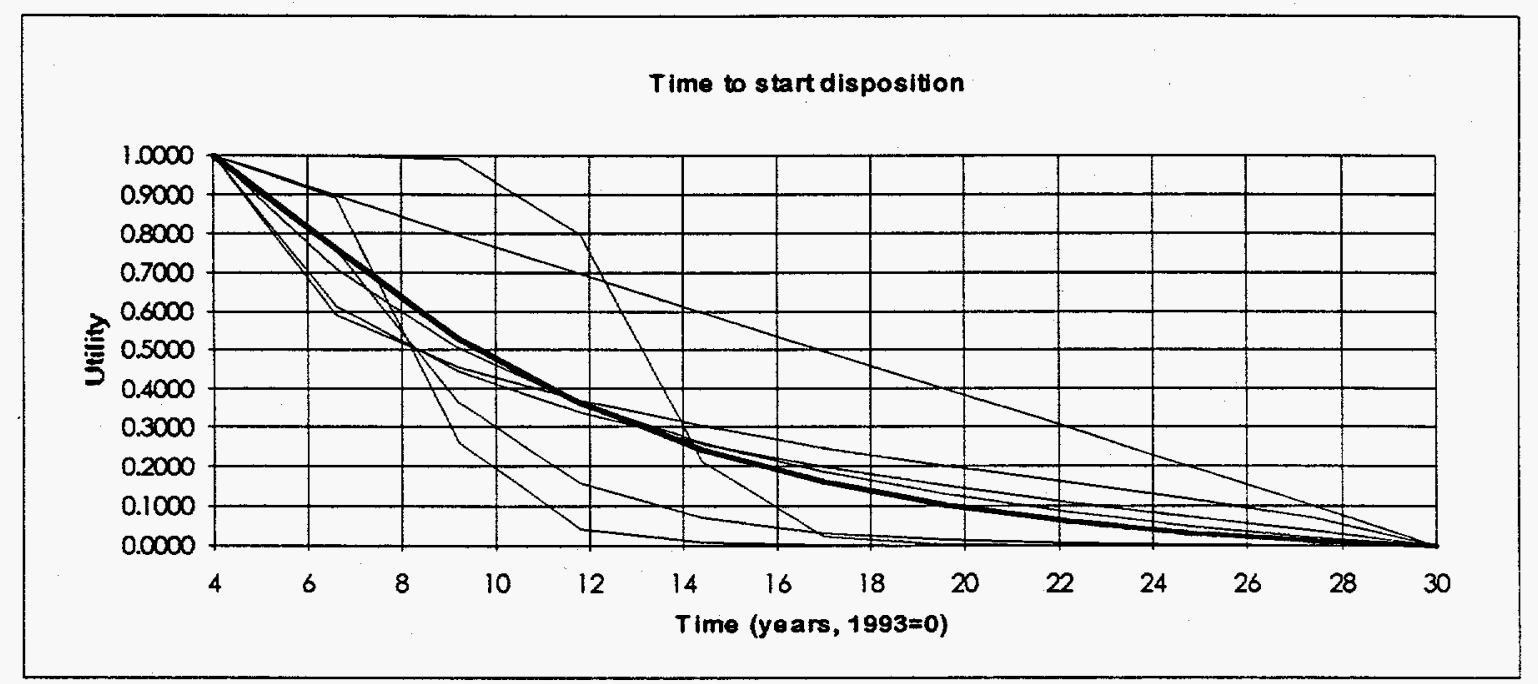

Time to complete disposition

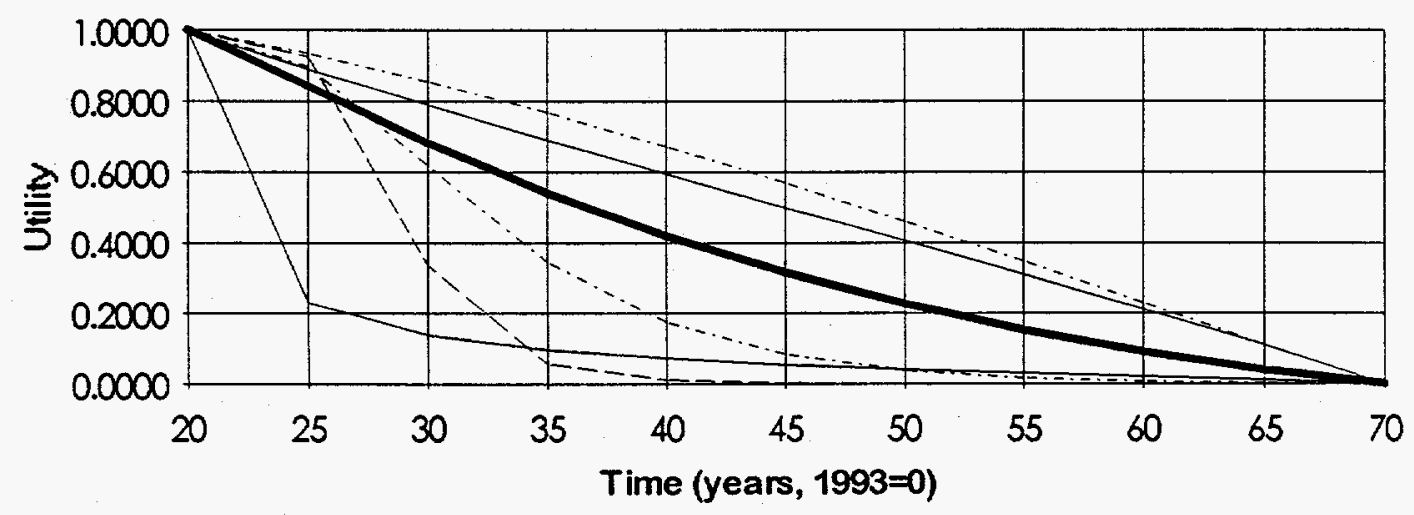



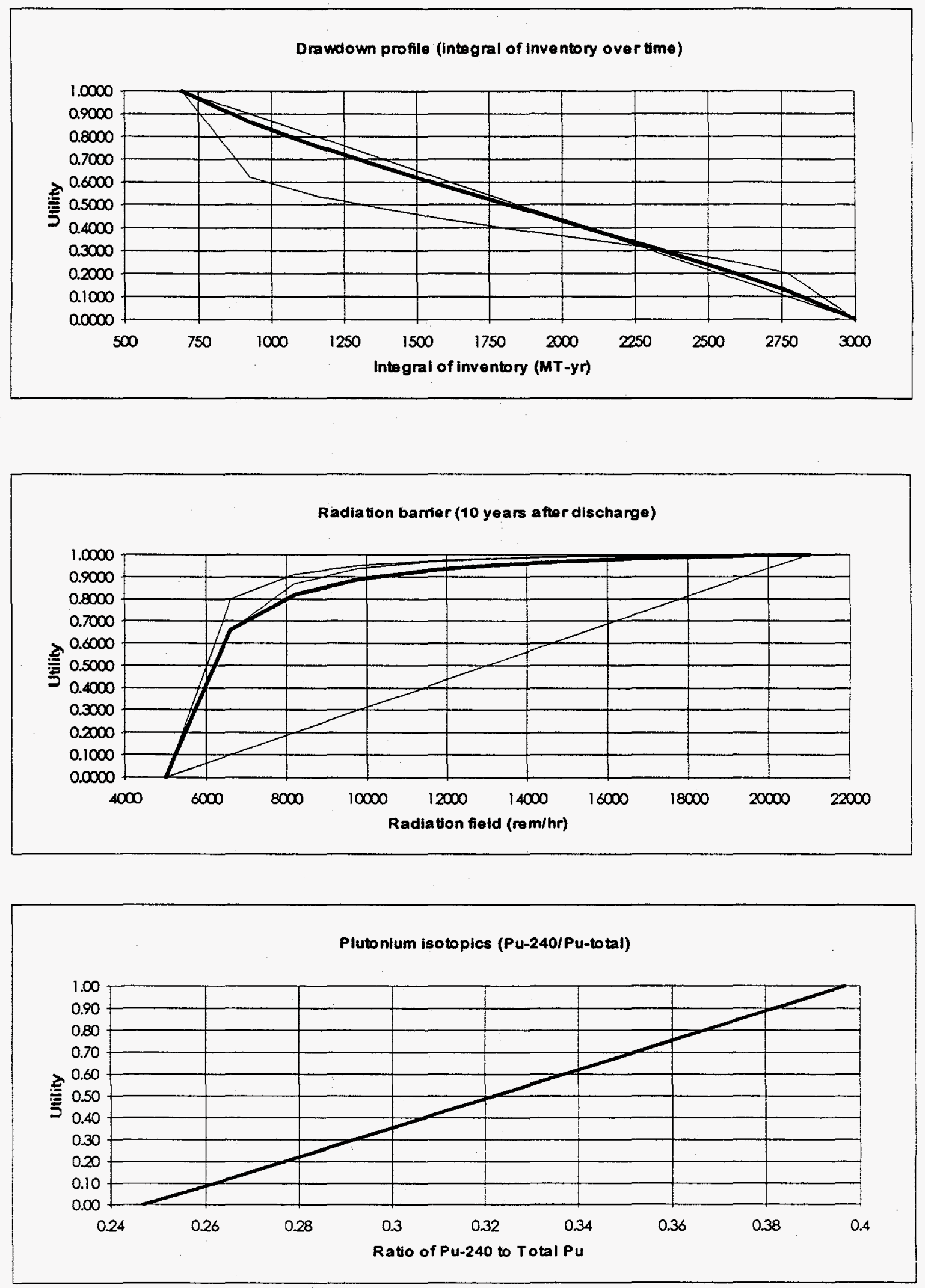

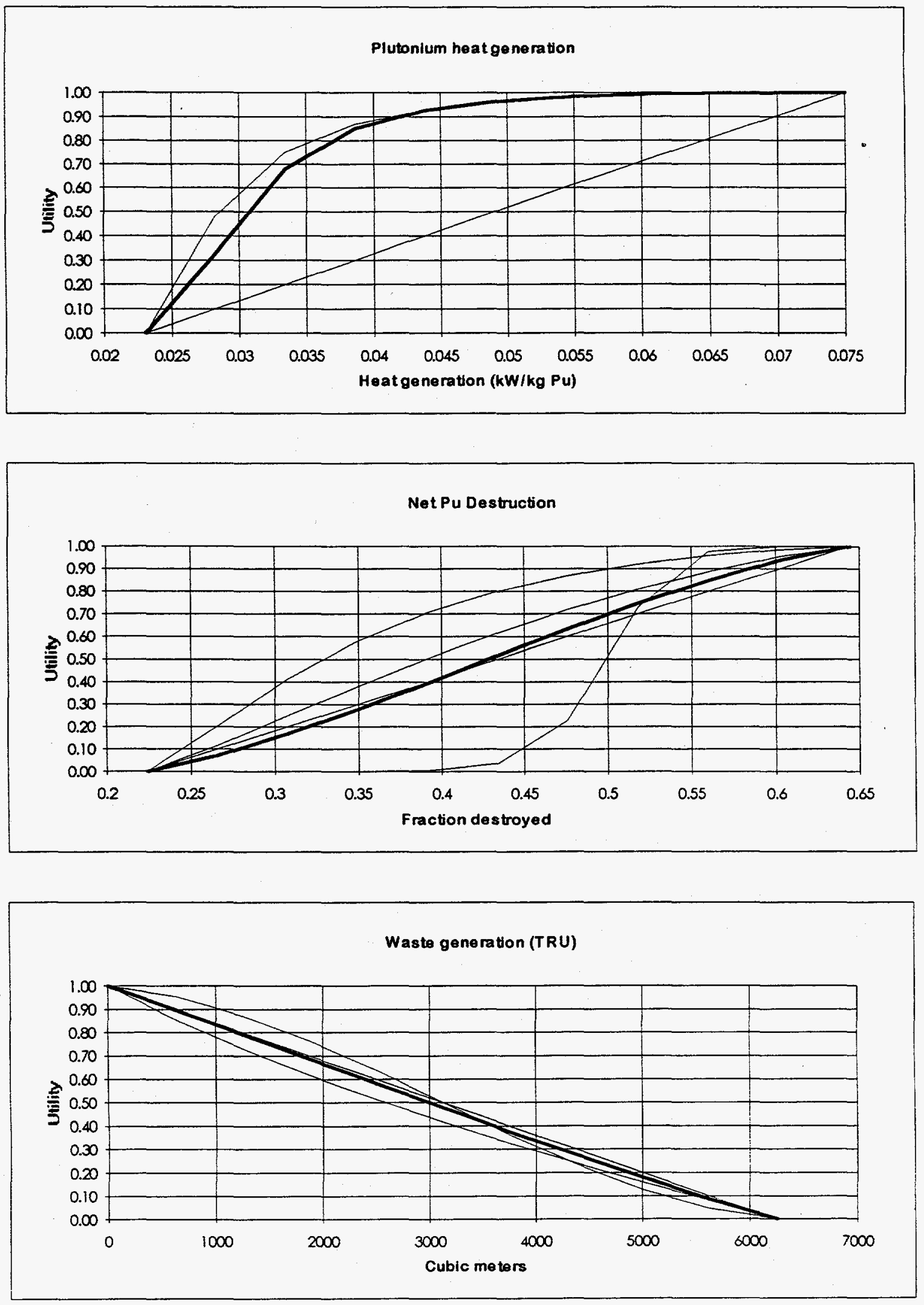

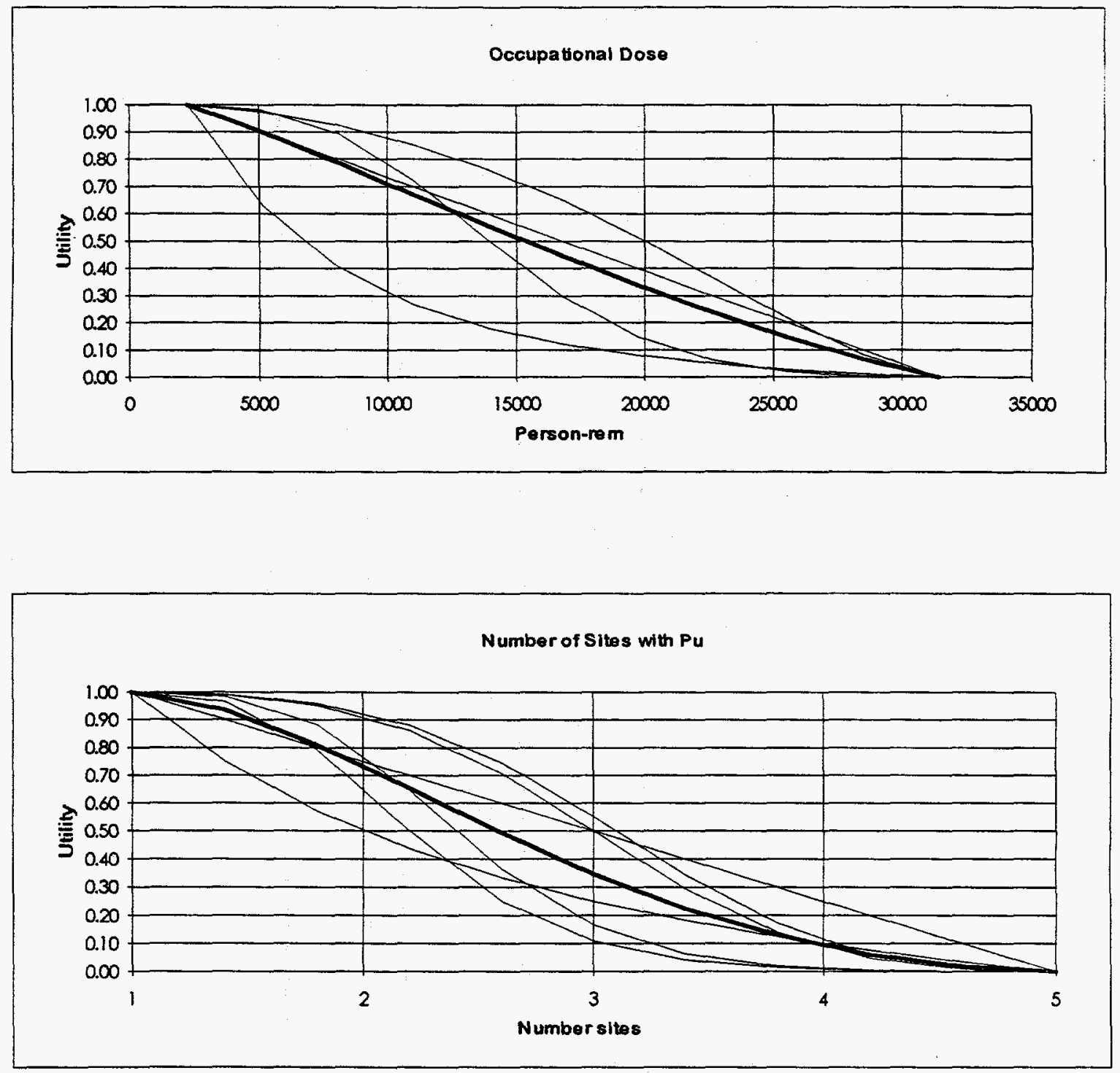

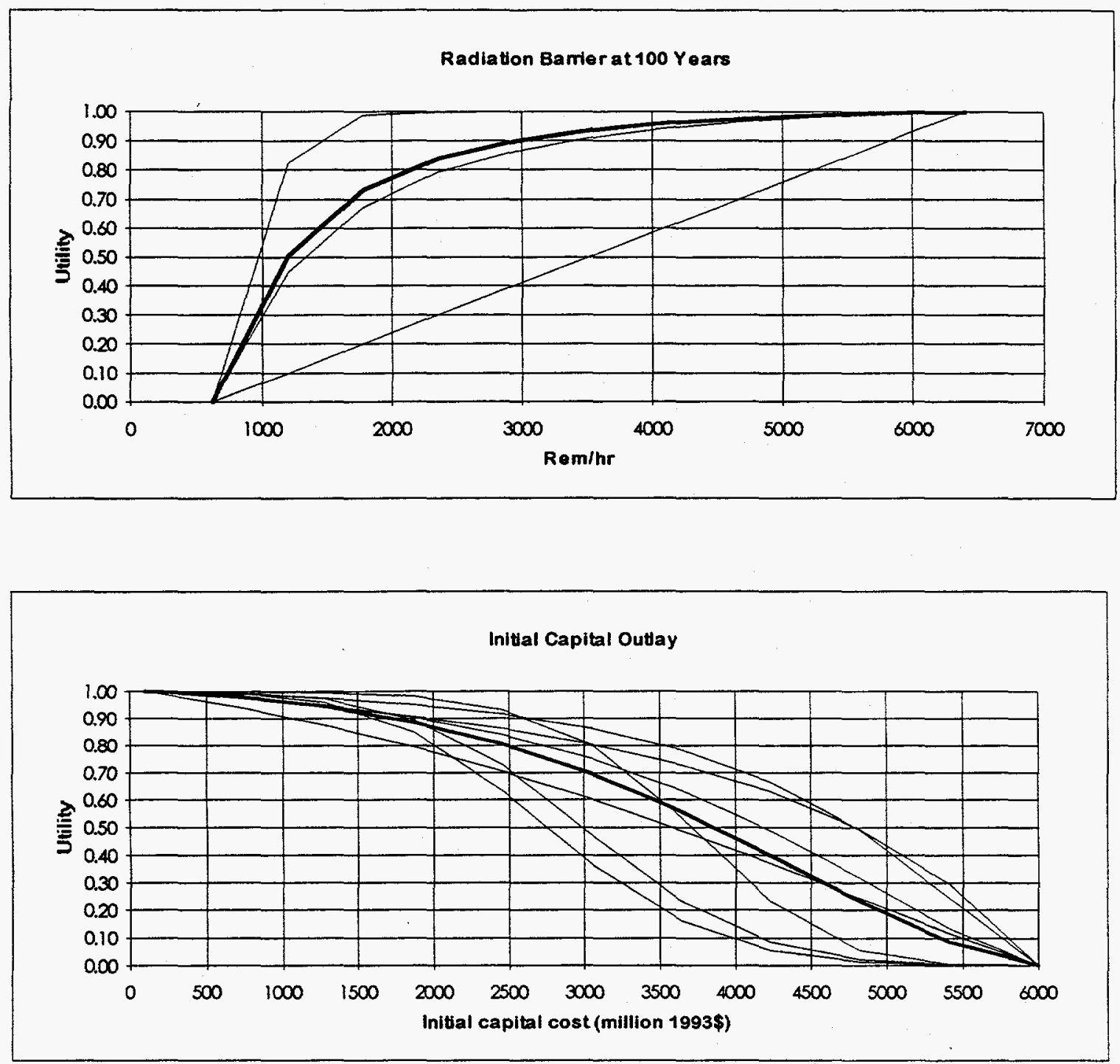


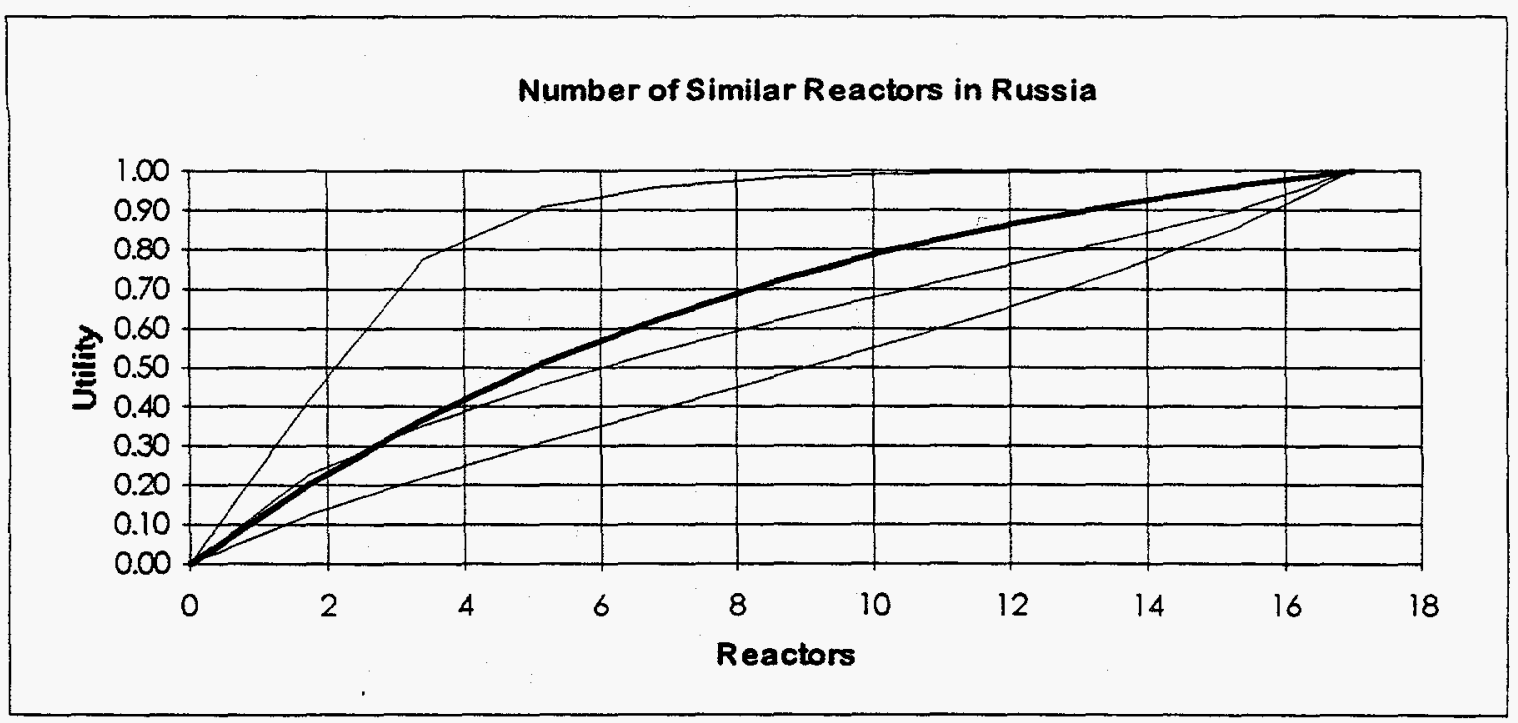

The parameters and corresponding utility function values for the seven reactor designs evaluated are shown in the two tables below. The existing CE design (EXCE) was not included in the evaluation because the data set was incomplete. Assumed values for the EXCE design are shown for illustration purposes only.

\section{Table A-1 Utility Function Values for Advanced Reactor Designs}

\begin{tabular}{|c|c|c|c|c|c|c|c|c|}
\hline \multicolumn{3}{|c|}{ Multiattribute U tility Function Assessment } & \multirow{2}{*}{\multicolumn{2}{|c|}{$\mathbf{W}$}} & \multirow{2}{*}{\multicolumn{2}{|c|}{$\mathrm{CE} / \mathrm{AB} \mathrm{B}$}} & & \\
\hline 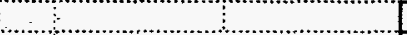 & GE & & & & & & \multicolumn{2}{|c|}{ GA-MHTGR } \\
\hline Utility Function & value & utiil & value & util & value & util. & value & util. \\
\hline 1 Life cycle cost: & 1123 & 0.6579 & 1413 & 0.6115 & 1213 & 0.6438 & 607 & 0.7315 \\
\hline 2Time to start disposition & 11.00 & 0.4069 & 11.00 & 0.4069 & 9.00 & 0.5457 & 14.00 & 0.2584 \\
\hline 3:Time to comple te disposition & 26.00 & 0.8081 & 26.00 & 0.8081 & 26.00 & 0.8081 & 26.00 & 0.8081 \\
\hline 4 Drawdown profile & 875 & 0.8877 & 894 & 0.8779 & 818 & 0.9182 & $996:$ & 0.8285 \\
\hline 5:Radiation barrier at 10 years & 16,000 & 0.9763 & 17,000 & 0.9826 & 63,484 & 0.9622 & 6,000 & 0.5367 \\
\hline 6 Isotopic suitability for weapons & 0.3374 & 0.6043 & 0.2535 & 0.0453 & 0.2488 & 0.0140 & 0,3440 & 0.6482 \\
\hline 7Thermal suitability for weapon & 0.031 & 0.5414 & 0.023 & 0.0000 & 0.025 & 0.0692 & 0.023 & 0.0000 \\
\hline 8 NetPudestruction & 0.3800 & 0.3627 & 0.2527 & 0.0455 & 0.2716 & 0.0842 & 0.6444 & 1.0000 \\
\hline 9: Waste generation (TRU) & 452 & 0.9278 & 450 & 0.9281 & 3629 & 0.3966 & 4.92 & 1.0000 \\
\hline 10 Occupational dose & 3257 & 0.9689 & 4270 & 0.9332 & 2240 & 1.0000 & 4632 & 0.9200 \\
\hline 11 Number of sites with $\mathrm{Pu}$ & 1 & 1.0000 & 1 & 1.0000 & 11 & 1.0000 & 1 & 1.0000 \\
\hline 12 Radiation barrier at 100 years & 1300 & 0.5601 & 1600 & 0.6798 & 6715.635 & 0.9980 & 620 & 0.0000 \\
\hline 13 Initial capizil outay & 4798 & 0.2401 & 4483.6 & 0.3282 & 5830.7 & 0.0137 & 5100 & 0.1595 \\
\hline 14 Number of similar reactors in R & 0 & 0.0000 & 17 & 1.0000 & o & 0.0000 & 0 & 0.0000 \\
\hline \multirow[t]{3}{*}{15 Tite 15} & 0 & 0.0000 & 0 & 0.0000 & 0 & 0.0000 & 0 & 0.0000 \\
\hline & UStatic: & $1.78 \mathrm{E}-01$ & \multirow{2}{*}{$\begin{array}{l}\text { UStatic: } \\
\text { UTotal: }\end{array}$} & $236 E-01$ & \multirow{2}{*}{$\begin{array}{l}\text { UStatic: } \\
\text { UTobl: }\end{array}$} & $9.76 \mathrm{E}-02$ & \multirow{2}{*}{$\begin{array}{l}\text { UStatic: } \\
\text { UT obl: }\end{array}$} & $1.59 \mathrm{E}-01$ \\
\hline & UT oul: & $5.13 \mathrm{E}-01$ & & $5.64 \mathrm{E}-01$ & & $4.65 \mathrm{E}-01$ & & $4.67 \mathrm{E}-01$ \\
\hline
\end{tabular}


Table A-2 Utility Function Values for Existing Reactors

\begin{tabular}{|c|c|c|c|c|c|c|c|c|}
\hline \multicolumn{3}{|c|}{ Multiattribute Utility Function Assessment } & \multirow{2}{*}{\multicolumn{2}{|c|}{ EXW }} & \multirow{2}{*}{\multicolumn{2}{|c|}{$\begin{array}{l} \\
\mathrm{EXCE}\end{array}$}} & \multirow{2}{*}{\multicolumn{2}{|c|}{$\frac{\vdots}{\mathrm{EXCAN}}$}} \\
\hline 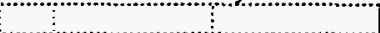 & \multicolumn{2}{|c|}{ EXGE } & & & & & & \\
\hline Utility Function & value & ubil & value & util. & value & util. & value & util. \\
\hline 1Life cycle cost & 1311 & 0.6282 & 1069 & 0.6661 & 1500 & 0.5968 & 1481 & 0.6001 \\
\hline 2 Time o start disposition & 7.50 & 0.6746 & 10.00 & 0.4717 & 6.00 & 0.8220 & 5.00 & 0.9221 \\
\hline 3 Time o complete disposition & 26.00 & 0.8081 & 26.00 & 0.8081 & 26.00 & 0.8081 & 26.00 & 0.8081 \\
\hline 4 Drawdown profile & 829 & 0.9124 & 867 & 0.8918 & 768 & 0.9471 & 760 & 0.9524 \\
\hline 5 Radiation barrier at 10 years & 17,800 & 0.9870 & 21,000 & 1.0000 & 49,500 & 0.9666 & 6,050 & 0.5499 \\
\hline 6 Isotopic suitability for weapons & 0.3729 & 0.8408 & 0.3090 & 0.4151 & 0.2467 & 0.0000 & 0.3968 & 1.0000 \\
\hline 7 Thermal suibability for weapon & 0.075 & 1.0000 & 0.060 & 09930 & 0.033 & 0.6668 & 0.035 & 0.7508 \\
\hline 8 NetPudestruction & 0.3648 & 03202 & 0.3260 & 02155 & 0.2235 & 0.0000 & 0.3380 & 0.2471 \\
\hline 9 Waste generation (TRU) & 6251 & 0.0000 & 464 & 0.9258 & 1058 & 0.8259 & 1720 & 0.7139 \\
\hline 10 Occupational dose & 31400 & 0.0000 & 10200 & 0.7026 & 2240 & 1.0000 & 4250 & 0.9340 \\
\hline 11 Nunber of sites with $\mathrm{Pu}$ & 2 & 0.7335 & 2 & 0.7335 & 2 & 0.7335 & 2 & 0.7335 \\
\hline 12 Radiation barrier at 100 years & 1400 & 0.6058 & 1720 & 0.7154 & 5200 & 0.9862 & 680 & 0.0568 \\
\hline 13 Initial capital outay & 1142 & 0.9547 & 853.9 & 09734 & 853.9 & 0.9734 & 486.7 & 0.9907 \\
\hline 14 Number of similar reactors in & o & 0.0000 & 17 & 1.0000 & 0 & 0.0000 & 0 & 0.0000 \\
\hline 15 T T ile 15 & 0 & 0.0000 & 0 & 0.0000 & D & 0.0000 & 0 & 0.0000 \\
\hline & UStatic: & $3.51 E-01$ & UStabic: & $4.08 \mathrm{E}-01$ & UStatic: & $3.66 \mathrm{E}-01$ & UStatic: & $3.73 \mathrm{E}-01$ \\
\hline 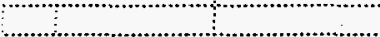 & UTotal: & $7.46 E-01$ & UT́obi: & $7.60 \mathrm{E}-01$ & UTotal: & $7.93 \mathrm{E}-01$ & UTotal: & $8.25 \mathrm{E}-01$ \\
\hline
\end{tabular}

The first four utility function values are baseline values that assume there are no delays and that the reactor performs at a $75 \%$ capacity factor. Utility function values for delays or different capacity factors are computed in the decision tree.

The values for utility functions five through fourteen are static, in that they are not affected by delays or different capacity factors. These values are summed, referenced as UStatic, and transmitted to the decision tree code where they are used without further modification. 


\title{
Appendix B - DPL Code for Decision Tree Model
}

\begin{abstract}
$/ / * * * * * * * * * * * * * * * * * * * * * * * * * * * * * * * * * * * * * * * * * * * * * * * * * * * * * * * * * * * * * * * * * * * * * * * * * *$
//Decision Tree Model for Pu Disposition with Advanced Reactors

// LPDS-034

// Tom Edmunds

// September 20, 1994
\end{abstract}

$/ / * * * * * * * * * * * * * * * * * * * * * * * * * * * * * * * * * * * * * * * * * * * * * * * * * * * * * * * * * * * * * * * * * * * * * * *$

// Link to spreadsheet for utility function assessments

string Excel_l="c:llweapdispllpullutilslibob2.xls";

// Global parameters

value InitStockpile=50000; $\quad / / \mathrm{kg} \mathrm{Pu}$

value DiscountRate=.04; / /per ORNL cost calculations

value $d=1 /(1+$ DiscountRate $)$;

value ElecPrice=.01919; $\quad / / 1993 \$$ per kwh in 1993

value ElecEsc $=.015$; $\quad$ //effective electrical energy price escalation

$/ /$ matches ORNL escalation curve in year 2020

value CapPay $=0.01 ; \quad / / 1993 \$$ per $\mathrm{kwh}$ does not escalate

value eps $=.000001$; $\quad / / u s e d$ to prevent divide by zero in utility fns.

value DelayCostFraction $=.20 ; \quad / / F r a c t i o n$ of ConsCost1 expended each year

value Gamma $=((1+$ DiscountRate $) /(1+$ ElecEsc $))-1$;

$/ /$ during construction delay

//equivalent discount factor for electricity

// price that takes into account discount and

$/ /$ escalation rates $(1+e) /(1+d)=1 /(1+$ Gamma $)$

//All ORNL cost data are in $1993 \$$

/Nendor schdules use 1994 as base year so add 1 year to convert to 1993 base year

// Parameters for advanced/evolutionary reactors:

// GE technology parameters

value GETimeLic $=18 / 12$;

/years from project start to construction permit

value GETimeConst=11-GETimeLic;

value GEInitCap $=4798$;

//years from const. permit to comm. ops. - ORNL

value GEConstCost $1=0.25 *$ GEInitCap;

value GEConstCost $2=0.75 *$ GEInitCap;

value GECoreInv $=6631$;

value GEElecCap $=2 * 1300 * 1000$;

// per ORNL spreadsheets

//costs prior to construction delay

//costs after delay

$/ / \mathrm{kg} \mathrm{Pu}$

$/ / k w$

value GENormalize $=0.973$;

//adjust so that multiple units represented

// by single equivalent unit

value GEOMCost=7752+1675-GEInitCap;//back out ORNL LEU ops costs and initial

// capital from total cost

value GEPuDispRate $=($ InitStockpile-GECoreInv)/(26-GETimeLic-GETimeConst):

$/ / \mathrm{kg}$ Pu/year at nominal capacity factor

// W technology parameters

value WTimeLic $=54 / 12$;

value WTimeConst=11-WTimeLic;

$/ /$ per ORNL

value WInitCap $=4484$;

value WConstCost $1=0.25 *$ WInitCap;

value WConstCost $2=0.75 *$ WInitCap;

value WCoreInv $=4081$; 
value WElecCap $=4 * 600 * 1000$;

value WNormalize $=0.989$;

value WOMCost $=8160+846$-WInitCap;

value WPuDispRate=(InitStockpile-WCoreInv)/(26-WTimeLic-WTimeConst);

// CE technology parameters

value $\mathrm{CETimeLic}=18 / 12$;

value CETimeConst=9-CETimeLic; //per ORNL

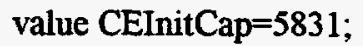

value $C E C$ onstCost $1=0.25 *$ CEInitCap;

value CEConstCost $2=0.75 * \mathrm{CEInitCap}$;

value CECoreInv $=6670$;

value $\mathrm{CEElecCap}=2 * 1250 * 1000$;

value $C E N o r m a l i z e=0.997$;

value CEOMCost $=9343+724-$ CEInitCap;

value CEPuDispRate=(InitStockpile-CECoreInv)/(26-CETimeLic-CETimeConst);

// GA technology parameters

value GATimeLic $=86 / 12$;

value GATimeConst=14-GATimeLic; //per ORNL

value GAInitCap=5100;

value GAConstCost $1=0.25 *$ GAInitCap;

value GAConstCost $2=0.75 *$ GAInitCap;

value GACoreInv $=634$;

value GAElecCap $=14 * 286 * 1000$; //GA plans to build 14 reactors

value GANormalize $=0.923$; //needed to represent 14 reactors by 1 equivalent reactor

value GAOMCost=9826+1931-GAInitCap;

value GAPuDispRate=(InitStockpile-GACoreInv)/(26-GATimeLic-GATimeConst);

// Parameters for existing reactors: $* * * * * * * * * * * * * * * * * * * * * * * * * * * * * * * * * * * * * * * * * * * * *$

// EXGE technology parameters

value EXGETimeLic=2; $\quad$ //Assumption

value EXGETimeConst=7.5-EXGETimeLic;

value EXGEInitCap $=1142$;

value EXGEConstCost $1=0.25 *$ EXGEInitCap;

value EXGEConstCost $2=0.75 *$ EXGEInitCap;

value EXGECoreInv=6631; $\quad$ //Assume same as advanced reactors

value EXGEElecCap $=0$;

//Assume existing plant output owned by utility

value EXGENormalize $=1.0$;

value EXGEOMCost=2310-999-EXGEInitCap;

value EXGEPuDispRate=(InitStockpile-EXGECoreInv)/(26-EXGETimeLic-EXGETimeConst);

// EXW technology parameters

value EXWTimeLic $=2$;

//Assumption

value EXWTimeConst=10-EXWTimeLic;

value EXWInitCap $=854$;

value EXWConstCost $1=0.25 *$ EXWInitCap;

value EXWConstCost $2=0.75 * E X W I n i t C a p$;

value EXWCoreInv $=4065$;

value EXWElecCap $=0$;

value EXWNormalize $=1.0$;

value EXWOMCost=1898-829-EXWInitCap;

value EXWPuDispRate=(InitStockpile-EXWCoreInv)/(26-EXWTimeLic-EXWTimeConst); 
// EXCE technology parameters - this reactor design excluded from analysis due to insufficient data value EXCETimeLic $=2$; //Assume same as EXW value EXCETimeConst=10-EXCETimeLic; //Assume same as EXW value EXCEInitCap $=1000$; //Assumption value EXCEConstCost $1=0.25 *$ EXCEInitCap; value EXCEConstCost2 $=0.75 *$ EXCEInitCap; value EXCECoreInv $=6670$; //Assume same as advanced CE value EXCEElecCap $=0$; value EXCENormalize $=1.0$; value EXCEOMCost=1898-829-EXCEInitCap; //Assume same as EXW value EXCEPuDispRate=(InitStockpile-EXCECoreInv)/(26-EXCETimeLic-EXCETimeConst);

// EXCAN technology parameters value EXCANTimeLic $=1$; value EXCANTimeConst=5-EXCANTimeLic; //Assumption value EXCANInitCap $=487$; value EXCANConstCost $1=0.25 *$ EXCANInitCap; value EXCANConstCost $2=0.75 *$ EXCANInitCap; value EXCANCoreInv=1448; value EXCANElecCap $=0$; value EXCANNormalize $=1.0$; value EXCANOMCost=1683-201-EXCANInitCap; value EXCANPuDispRate=(InitStockpile-EXCANCoreInv)/(26-EXCANTimeLic-EXCANTimeConst);

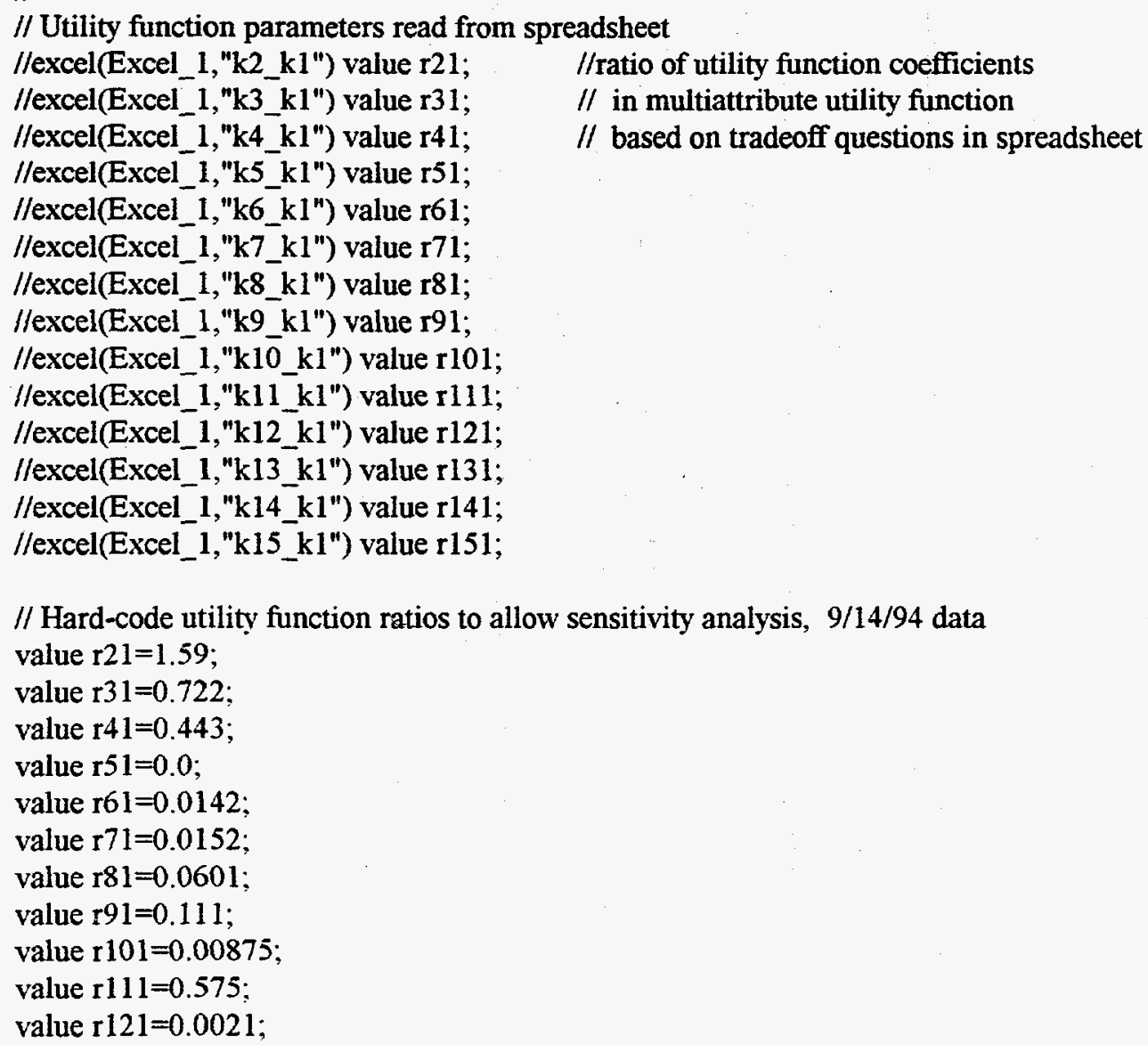

// Hard-code utility function ratios to allow sensitivity analysis, 9/14/94 data value $\mathrm{r} 21=1.59$ 
value $\mathrm{r} 131=2.01$

value $\mathrm{r} 141=0.254$

value $\mathrm{r} 151=0.0000$;

//computation of multiattribute utility function coefficients given ratios

value $\mathrm{k} 1=1 /(1+\mathrm{r} 21+\mathrm{r} 31+\mathrm{r} 41+\mathrm{r} 51+\mathrm{r} 61+\mathrm{r} 71+\mathrm{r} 81+\mathrm{r} 91+\mathrm{r} 101+\mathrm{r} 111+\mathrm{r} 121+\mathrm{r} 131+\mathrm{r} 141+\mathrm{r} 151)$;

value $\mathrm{k} 2 \mathrm{k} 1 \mathrm{k}^{\mathrm{r}} \mathrm{r} 21$;

value $\mathrm{k} 3=\mathrm{k} 1 * \mathrm{r} 31$

value $\mathrm{k} 4=\mathrm{k} 1 * \mathrm{r} 41$

value $\mathrm{k} 5=\mathrm{kl}{ }^{*} \mathrm{r} 51$;

value $\mathrm{k} 6=\mathrm{kl} \mathbf{r}^{*} \mathrm{r} 61$;

value $\mathrm{k} 7=\mathrm{k} 1 * \mathrm{r} 71$

value $\mathrm{k} 8=\mathrm{k} 1{ }^{*} \mathrm{r} 81$

value $\mathrm{k} 9=\mathrm{kl}{ }^{*} \mathrm{r} 91$

value $\mathrm{k} 10=\mathrm{k} 1 * \mathrm{r} 101$

value $\mathrm{k} 11=\mathrm{k} 1 * \mathrm{r} 111$;

value $\mathrm{k} 12=\mathrm{k} 1 * \mathrm{r} 121$;

value $\mathrm{k} 13=\mathrm{k} 1{ }^{*} \mathrm{r} 131$

value $\mathrm{k} 14=\mathrm{k} 1 *_{\mathrm{r} 141}$;

value $\mathrm{k} 15=\mathrm{k} 1 * \mathrm{r} 151$

$/ /$ read in coefficients for first 4 utility functions

// $1=$ Cost

excel(Excel_1,"c_1") value cl;

excel(Excel_1,"b_l") value bl;

excel(Excel_1,"worst1") value worst1;

excel(Excel_1,"best1") value best1;

// 2=Time to start

excel(Excel_1,"c_2") value c2;

excel(Excel_1,"b_2") value b2;

excel(Excel_1,"worst2") value worst2;

excel(Excel_1,"best2") value best2;

// 3=Time to complete

excel(Excel_1,"c_3") value c3;

excel(Excel_1,"b_3") value b3;

excel(Excel_1,"worst3") value worst3;

excel(Excel_1,"best3") value best3;

// 4=MT-yr

excel(Excel_1,"c_4") value c4;

excel(Excel_1,"b_4") value b4;

excel(Excel_1,"worst4") value worst4;

excel(Excel_1,"best4") value best4;

I/Utility function values for attributes that are independent of time and cost

//GE static utility function values

excel(Excel_1,"UGE5") value UGE5;

excel(Excel_1,"UGE6") value UGE6;

excel(Excel_1,"UGE7") value UGE7;

excel(Excel_1,"UGE8") value UGE8;

excel(Excel_1,"UGE9") value UGE9;

excel(Excel_1,"UGE10") value UGE10;

excel(Excel_1,"UGE11") value UGE11;

excel(Excel_1,"UGE12") value UGE12;

excel(Excel_1,"UGE13") value UGE13; 
excel(Excel_1,"UGE14") value UGE14; excel(Excel_1,"UGE15") value UGE15; I/W static utility function values excel(Excel_1,"UW5") value UW5; excel(Excel_1, "UW6") value UW6; excel(Excel_1,"UW7") value UW7; excel(Excel_1, "UW8") value UW8; excel(Excel_1,"UW9") value UW9; excel(Excel_1,"UW10") value UW10; excel(Excel_1,"UW11") value UW11; excel(Excel_1,"UW12") value UW12; excel(Excel_1,"UW13") value UW13; excel(Excel_1,"UW14") value UW14; excel(Excel_1,"UW15") value UW15; //CE static utility function values excel(Excel_1,"UCE5") value UCE5; excel(Excel_1,"UCE6") value UCE6; excel(Excel_1,"UCE7") value UCE7; excel(Excel_1,"UCE8") value UCE8; excel(Excel_1,"UCE9") value UCE9; excel(Excel_1,"UCE10") value UCE10; excel(Excel_1,"UCE11") value UCE11; excel(Excel_1,"UCE12") value UCE12; excel(Excel_1,"UCE13") value UCE13; excel(Excel_1,"UCE14") value UCE14; excel(Excel_1,"UCE15") value UCE15; //GA static utility function values excel(Excel_1,"UGA5") value UGA5; excel(Excel_1,"UGA6") value UGA6; excel(Excel_1,"UGA7") value UGA7; excel(Excel_1,"UGA8") value UGA8; excel(Excel_1,"UGA9") value UGA9; excel(Excel_1,"UGA10") value UGA10; excel(Excel_1,"UGA11") value UGA11; excel(Excel_1,"UGA12") value UGA12; excel(Excel_1,"UGA13") value UGA13; excel(Excel_1,"UGA14") value UGA14; excel(Excel_1,"UGA15") value UGA15; //EXGE static utility function values excel(Excel_1,"UEXGE5") value UEXGE5; excel(Excel_1,"UEXGE6") value UEXGE6; excel(Excel_1,"UEXGE7") value UEXGE7; excel(Excel_1,"UEXGE8") value UEXGE8; excel(Excel_1,"UEXGE9") value UEXGE9; excel(Excel_1,"UEXGE10") value UEXGE10; excel(Excel_1,"UEXGE11") value UEXGE11; excel(Excel_1,"UEXGE12") value UEXGE12; excel(Excel_1,"UEXGE13") value UEXGE13; excel(Excel_1,"UEXGE14") value UEXGE14; excel(Excel_1,"UEXGE15") value UEXGE15; /EXW static utility function values excel(Excel_1,"UEXW5") value UEXW5; excel(Excel_1,"UEXW6") value UEXW6; excel(Excel_1,"UEXW7") value UEXW7; 
excel(Excel_1,"UEXW8") value UEXW8; excel(Excel_1,"UEXW9") value UEXW9; excel(Excel_1,"UEXW10") value UEXW10; excel(Excel_1,"UEXW11") value UEXW11; excel(Excel_1,"UEXW12") value UEXW12; excel(Excel_1,"UEXW13") value UEXW13; excel(Excel_1,"UEXW14") value UEXW14; excel(Excel_1,"UEXW15") value UEXW15; /EXCE static utility function values excel(Excel_1,"UEXCE5") value UEXCE5; excel(Excel_1,"UEXCE6") value UEXCE6; excel(Excel_1,"UEXCE7") value UEXCE7; excel(Excel_1,"UEXCE8") value UEXCE8; excel(Excel_1,"UEXCE9") value UEXCE9; excel(Excel_1,"UEXCE10") value UEXCE10; excel(Excel_1,"UEXCE11") value UEXCE11; excel(Excel_1,"UEXCE12") value UEXCE12; excel(Excel_1,"UEXCE13") value UEXCE13; excel(Excel_1,"UEXCE14") value UEXCE14; excel(Excel_1,"UEXCE15") value UEXCE15; //EXCAN static utility function values excel(Excel_1,"UEXCAN5") value UEXCAN5; excel(Excel_1,"UEXCAN6") value UEXCAN6; excel(Excel_1,"UEXCAN7") value UEXCAN7; excel(Excel_1,"UEXCAN8") value UEXCAN8; excel(Excel_1,"UEXCAN9") value UEXCAN9; excel(Excel_1,"UEXCAN10") value UEXCAN10; excel(Excel_1,"UEXCAN11") value UEXCAN11; excel(Excel_1,"UEXCAN12") value UEXCAN12; excel(Excel_1,"UEXCAN13") value UEXCAN13; excel(Excel_1,"UEXCAN14") value UEXCAN14; excel(Excel_1,"UEXCAN15") value UEXCAN15;

//Computation of fixed utility value UGEFixed=k5*UGE5+k6*UGE6+k7*UGE7+k8*UGE8+k9*UGE9+k10*UGE10 +k11*UGE11+k12*UGE12+k13*UGE13+k14*UGE14+k15*UGE15; value UWFixed $=\mathrm{k} 5 * \mathrm{UW} 5+\mathrm{k} 6 * \mathrm{UW6}+\mathrm{k} 7 * \mathrm{UW} 7+\mathrm{k} 8 * \mathrm{UW} 8+\mathrm{k} 9 * \mathrm{UW} 9+\mathrm{k} 10 * \mathrm{UW} 10$ $+k 11 * U W 11+k 12 * U W 12+k 13 * U W 13+k 14 * U W 14+k 15 * U W 15$; value UCEFixed $=k 5 * U C E 5+k 6 * U C E 6+k 7 * U C E 7+k 8 * U C E 8+k 9 * U C E 9+k 10 * U C E 10$ $+\mathrm{k} 11 * \mathrm{UCE} 11+\mathrm{k} 12 * \mathrm{UCE} 12+\mathrm{k} 13 * \mathrm{UCE} 13+\mathrm{k} 14 * \mathrm{UCE} 14+\mathrm{k} 15 * \mathrm{UCE} 15$ value UGAFixed $=k 5 * U G A 5+k 6 * U G A 6+k 7 * U G A 7+k 8 * U G A 8+k 9 * U G A 9+k 10 * U G A 10$ $+\mathrm{k} 11 * \mathrm{UGA} 11+\mathrm{k} 12 * \mathrm{UGA} 12+\mathrm{k} 13 * \mathrm{UGA} 13+\mathrm{k} 14 * \mathrm{UGA} 14+\mathrm{k} 15 * \mathrm{UGA} 15$ value UEXGEFixed $=$

$\mathrm{k} 5 *$ UEXGE5+k6*UEXGE6+k7*UEXGE7+k8*UEXGE8+k9*UEXGE9+k10*UEXGE 10 +k11*UEXGE11+k12*UEXGE12+k13*UEXGE13+k14*UEXGE14+k15*UEXGE15; value UEXWFixed $=\mathrm{k} 5 *$ UEXW5+k6*UEXW6+k7*UEXW7+k8*UEXW8+k9*UEXW9+k $10 *$ UEXW 10 +k11*UEXW11+k12*UEXW12+k13*UEXW13+k14*UEXW14+k15*UEXW15; value UEXCEFixed $=$ k5*UEXCE5+k6*UEXCE6+k7*UEXCE7+k8*UEXCE8+k9*UEXCE9+k10*UEXCE10 +k11*UEXCE11+k12*UEXCE12+k13*UEXCE13+k14*UEXCE14+k15*UEXCE15; value UEXCANFixed= $\mathrm{k} 5 *$ UEXCAN5+k6*UEXCAN6+k $7 *$ UEXCAN7+k8*UEXCAN8+k9*UEXCAN9 $+\mathrm{k} 10 *$ UEXCAN $10+\mathrm{k} 11 *$ UEXCAN $11+\mathrm{k} 12 *$ UEXCAN12+k13*UEXCAN 13 
+k14*UEXCAN14+k15*UEXCAN15;

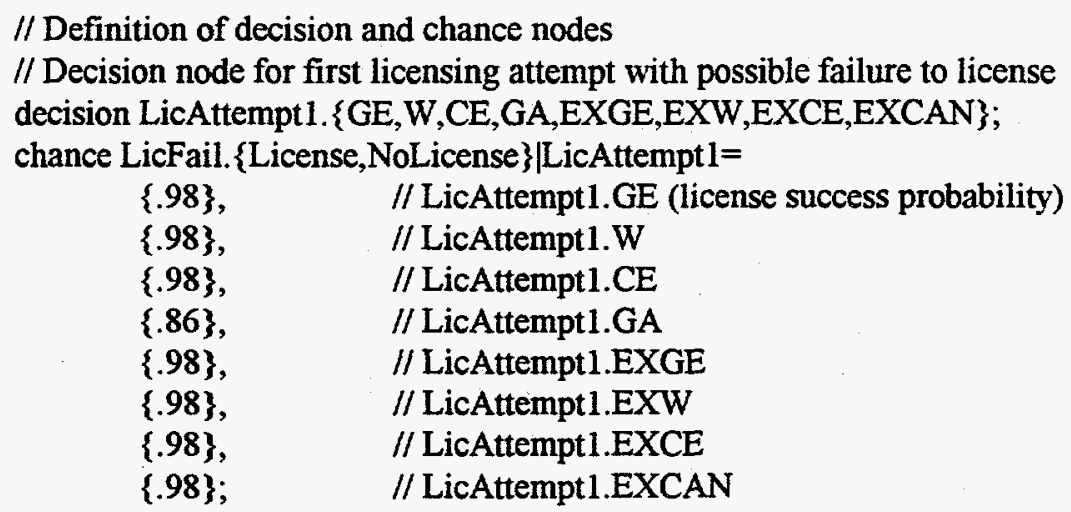

//GE chance nodes $* * * * * * * * * * * * * * * * * * * * * * * * * *$

// Licensing delay for GE given licensing is successful

chance GELicDelay. $\{$ None,Delay_2_yr,Delay_4_yr,Delay_6_yr $\}=\{.55 / .98, .23 / .98, .13 / .98\}=$,
0 ,
// GELicDelay.None
2 ,
// GELicDelay.Delay_2_yr
4
// GELicDelay.Delay_4_yr
6
// GELicDelay.Delay_6 yr

// Construction delay for GE

// (assumed to occur after ConstCostl is expended)

chance GEConstDelay. $\{$ None,Delay_2_yr,Delay_4_yr,Delay_6_yr $\}=\{.48,28, .19\}=$,

$\begin{array}{ll}0, & \text { // GEConstDelay.None } \\ 2, & \text { // GEConstDelay.Delay_2_yr } \\ 4, & \text { // GEConstDelay.Delay_4_yr } \\ 6 ; & \text { // GEConstDelay.Delay_6_yr }\end{array}$

// Capacity factor for GE

chance GECapFact. $\{$ Cap55, Cap65,Cap75,Cap85 $\}=\{.1,28, .5\}=$,
.55 ,
.65 ,
// GECapFact.Cap55
.75 ,
// GECapFact.Cap65
85
// GECapFact.Cap75
// GECapFact.Cap85

//W chance nodes $* * * * * * * * * * * * * *$

//Licensing delay given $\mathrm{W}$ is licensed

chance WLicDelay. $\{$ None,Delay_2_yr,Delay_4_yr,Delay_6_yr $\}=\{.4 / .98, .35 / .98, .15 / .98\}=$,
0 ,
// WLicDelay.None
2, // WLicDelay.Delay_2_yr
4, // WLicDelay.Delay_4_yr
6; $\quad$ // WLicDelay.Delay_6_yr

//Construction delay for $\mathrm{W}$

chance WConstDelay. $\{$ None,Delay_2_yr,Delay_4_yr,Delay_6_yr $\}=\{.4, .35, .19\}=$,
0 ,
// WConstDelay.None
2, //WConstDelay.Delay_2_yr
4, //WConstDelay.Delay_4_yr
6; $\quad$ // WConstDelay.Delay_6_yr 
//Capacity factor for W

chance WCapFact. $\{$ Cap55,Cap65, Cap75,Cap85 $\}=\{.1,28, .5\}=$,

$\begin{array}{ll}.55, & \text { // WCapFact.Cap55 } \\ .65, & \text { // WCapFact.Cap65 } \\ .75, & \text { // WCapFact.Cap75 } \\ .85 ; & \text { // WCapFact.Cap85 }\end{array}$

//CE chance nodes $* * * * * * * * * * * * * * * *$

//Licensing delay for $\mathrm{CE}$

chance CELicDelay. $\{$ None,Delay_2_yr,Delay_4_yr,Delay_6_yr $\}=\{.55 / .98, .23 / .98, .13 / .98\}=$,

$\begin{array}{ll}0, & \text { // CELicDelay.None } \\ 2, & \text { // CELicDelay.Delay_2_yr } \\ 4, & \text { // CELicDelay.Delay_4_yr } \\ 6 ; & \text { // CELicDelay.Delay_6_yr }\end{array}$

//Construction delay for $\mathrm{CE}$

chance CEConstDelay. $\{$ None,Delay_2_yr,Delay_4_yr,Delay_6_yr $\}=\{.48,28,19\}=$

$\begin{array}{ll}0, & \text { // CEConstDelay.None } \\ 2, & \text { // CEConstDelay.Delay_2_yr } \\ 4, & \text { // CEConstDelay.Delay_4_yr } \\ 6 ; & \text { // CEConstDelay.Delay_6_yr }\end{array}$

//Capacity factor for CE

chance CECapFact. $\{$ Cap55, Cap65, Cap75,Cap85 $\}=\{.1,29, .5\}=$,
.55
// CECapFact.Cap55
.65 ,
// CECapFact.Cap65
.75 ,
// CECapFact.Cap75
.85 ;
// CECapFact.Cap85

//GA chance nodes $* * * * * * * * * * * * * * * * * * * * *$

//Licensing delay for GA

chance GALicDelay. $\{$ None,Delay_2_yr,Delay_4_yr,Delay_6_yr $\}=\{.20 / .86, .25 / .86,30 / .86\}=$,

$\begin{array}{ll}0, & \text { // GALicDelay.None } \\ 2, & \text { // GALicDelay.Delay_2_yr } \\ 4, & \text { // GALicDelay.Delay_4_yr } \\ 6 ; & \text { // GALicDelay.Delay_6_yr }\end{array}$

//Construction delay for GA

chance GAConstDelay. $\{$ None,Delay_2_yr,Delay_4_yr,Delay_6_yr $\}=\{, 28,4, .23\}=$,
0 ,
// GAConstDelay. None
2, // GAConstDelay.Delay_2 yr
4, // GAConstDelay.Delay_4yr
6; $\quad$ // GAConstDelay.Delay_6 yr

//Capacity factor for GA

chance GACapFact. $\{$ Cap55,Cap65, Cap75, Cap85 $\}=\{.22, .17, .5\}=$,

.55 ,

.65 ,

// GACapFact.Cap55

// GACapFact.Cap65

.75

// GACapFact.Cap75

.85

// GACapFact.Cap85

//EXGE chance nodes

// Licensing delay for EXGE

chance EXGELicDelay. $\{$ None,Delay_2_yr,Delay_4_yr,Delay_6_yr $\}=\{.70 / 1,30 / 1,0 / 1\}=$, 


$$
\begin{array}{ll}
0, & \text { // EXGELicDelay.None } \\
2, & \text { // EXGELicDelay.Delay_2yr } \\
4, & \text { // EXGELicDelay.Delay_4yr } \\
6 ; & \text { // EXGELicDelay.Delay_6_yr }
\end{array}
$$

// Construction delay for EXGE (assumed to occur after ConstCostl is expended on first reactor) chance EXGEConstDelay. $\{$ None,Delay_2_yr,Delay_4_yr,Delay_6_yr $\}=\{.9,1,0\}=$,

$\begin{array}{ll}0, & \text { // EXGEConstDelay.None } \\ 2, & \text { //EXGEConstDelay.Delay_2_yr } \\ 4, & \text { //EXGEConstDelay.Delay_4_yr } \\ 6 ; & \text { // EXGEConstDelay.Delay_6_yr }\end{array}$

// Capacity factor for EXGE

chance EXGECapFact. $\{$ Cap55,Cap65,Cap75,Cap85 $\}=\{.1, .28, .5\}=$,

.55, //EXGECapFact.Cap55

65, // EXGECapFact.Cap65

.75, //EXGECapFact.Cap75

.85; //EXGECapFact.Cap85

//EXW chance nodes

$/ /$ Licensing delay given EXW is licensed

chance EXWLicDelay. $\{$ None,Delay_2_yr,Delay_4_yr,Delay_6_yr $\}=\{.70 / 1,30 / 1,0 / 1\}=$,
0 ,
// EXWLicDelay.None
2, //EXWLicDelay.Delay_2_yr
4, //EXWLicDelay.Delay_4_yr
6; $\quad$ //EXWLicDelay.Delay_6_yr

//Construction delay for EXW

chance EXWConstDelay. $\{$ None,Delay_2_yr,Delay_4_yr,Delay_6_yr $\}=\{.9,1,0\}=$

$\begin{array}{ll}0, & / / \text { EXWConstDelay.None } \\ 2, & \text { // EXWConstDelay.Delay_2_yr } \\ 4, & \text { // EXWConstDelay.Delay_4_yr } \\ 6 ; & \text { // EXWConstDelay.Delay_6_yr }\end{array}$

//Capacity factor for EXW

chance EXWCapFact. $\{$ Cap55,Cap65,Cap75,Cap85 $\}=\{.1,28, .5\}=$,

.55, // EXWCapFact.Cap55

.65, //EXWCapFact.Cap65

.75, //EXWCapFact.Cap75

.85; // EXWCapFact.Cap85

//EXCE chance nodes *

//Licensing delay given EXCE is licensed

chance EXCELicDelay. $\{$ None,Delay_2_yr,Delay_4_yr,Delay_6_yr $\}=\{.60 / 1,40 / 1,0 / 1\}=$,

0 , // EXCELicDelay.None

2 , //EXCELicDelay.Delay_2_yr

4, //EXCELicDelay.Delay_4_yr

6; $\quad / /$ EXCELicDelay.Delay_6_yr

//Construction delay for EXCE

chance EXCEConstDelay. $\{$ None,Delay_2_yr,Delay_4_yr,Delay_6_yr $\}=\{.9,1,0\}=$,

0 ,

// EXCEConstDelay.None

2, // EXCEConstDelay.Delay_2_yr

4, // EXCEConstDelay.Delay_4_yr 
6;

// EXCEConstDelay.Delay_6_yr

//Capacity factor for EXCE

chance EXCECapFact. $\{$ Cap55, Cap65, Cap75, Cap85 $\}=\{.1,29, .5\}=$,

$.55, \quad / /$ EXCECapFact.Cap55

65, //EXCECapFact.Cap65

.75, // EXCECapFact.Cap75

.85; // EXCECapFact.Cap85

//EXCAN chance nodes **************

//Licensing delay given EXCAN is licensed

chance EXCANLicDelay. $\{$ None,Delay_2_yr,Delay_4_yr,Delay_6_yr $\}=\{.75 / 1, .25 / 1,0 / 1\}=$,

0 , // EXCANLicDelay.None

2, // EXCANLicDelay.Delay_2_yr

4, // EXCANLicDelay.Delay_4_yr

6; $\quad$ // EXCANLicDelay.Delay_6_yr

//Construction delay for EXCAN

chance EXCANConstDelay. $\{$ None,Delay_2_yr,Delay_4_yr,Delay_6_yr $\}=\{.9,1,0\}=$,
0 ,
// EXCANConstDelay.None
2 ,
// EXCANConstDelay.Delay_2 yr
4 ,
// EXCANConstDelay.Delay_4_yr
6
// EXCANConstDelay.Delay_6_yr

//Capacity factor for EXCAN

chance EXCANCapFact. $\{$ Cap55, Cap65,Cap75,Cap85 $\}=\{.1, .2, .5\}=$,

.55 ,

.65 ,

.75 ,

.85

// EXCANCapFact.Cap55

// EXCANCapFact.Cap65

// EXCANCapFact.Cap75

// EXCANCapFact.Cap85

/Time spent on first licensing attempt in years * $/ /$ (second licensing effort started after this time) value LicFailTime|LicAttempt1, LicFail=

// LicAttempt1.GE

0 ,

6, // LicFail.NoLicense

/I LicFail.License

$0, \quad / / \quad$ LicFail.License

6, $/ / \quad$ LicFail.NoLicense

$0, \quad / /$ LicAttemptl.CE

6, // LicFail.NoLicense

// LicAttempt1.GA

$0, \quad / / \quad$ LicFail.License

6, // LicFail.NoLicense

0, $/ /$ LicAttemptl.EXGE

3, // LicFail.NoLicense

$0, \quad / /$ LicAttempt1.EXW

3, // LicFail.NoLicense

0, $/ / \quad$ LicFail.License 


\begin{tabular}{|c|c|}
\hline 3 , & $\begin{array}{l}/ / \quad \text { LicFail.NoLicense } \\
/ / \text { LicAttemptl.EXCAN }\end{array}$ \\
\hline 0 , & LicFail.License \\
\hline 3; & LicFail.NoLicense \\
\hline
\end{tabular}

//cost expended on licensing attempt, million \$ value LicFailCost|LicAttempt 1, LicFail $=$

\begin{tabular}{|c|c|c|c|}
\hline \multirow{4}{*}{$\begin{array}{l}0, \\
1000\end{array}$} & \multirow{4}{*}{$/ 1$} & // LicAttemptl.GE & \multirow[t]{3}{*}{ Assume spend $\$ 1$ billion in license attempt } \\
\hline & & LicFail.License & \\
\hline & & LicFail.NoLicense & \\
\hline & & // LicAttempt1.W & \\
\hline 0 & & $/ / \quad$ LicFail.License & \\
\hline 1000 & $/ /$ & $\begin{array}{l}\text { LicFail.NoLicense } \\
\text { // LicAttempt1.CE }\end{array}$ & \\
\hline 0 & & // LicFail.License & \\
\hline 1000, & $/ /$ & $\begin{array}{l}\text { LicFail.NoLicense } \\
\text { // LicAttemptl.GA }\end{array}$ & \\
\hline 0 & & $/ / \quad$ LicFail.License & \\
\hline 1000 , & $/ 1$ & $\begin{array}{l}\text { LicFail.NoLicense } \\
\text { // LicAttempt1.EXGE }\end{array}$ & \\
\hline 0 & & LicFail.License & \\
\hline 500 & $/ /$ & $\begin{array}{l}\text { LicFail.NoLicense } \\
\text { // LicAttempt1.EXW }\end{array}$ & Assume spend $\$ 500$ million in license attempt \\
\hline 0 , & & LicFail.License & \\
\hline 500 & $/ 1$ & $\begin{array}{l}\text { LicFail.NoLicense } \\
\text { // LicAttempt1.EXCE }\end{array}$ & \\
\hline 0 & & LicFail.License & \\
\hline 500 & $/ /$ & $\begin{array}{l}\text { LicFail.NoLicense } \\
\text { // LicAttemptl.EXCAN }\end{array}$ & \\
\hline $\begin{array}{l}0 \\
500\end{array}$ & $/ /$ & $\begin{array}{l}\text { // LicFail.License } \\
\text { LicFail.NoLicense }\end{array}$ & \\
\hline
\end{tabular}

/MOX fuel plant delay

//CANDU MOX fuel plant delay

chance CANFuelDelay. $\{$ None,Delay_2_yr,Delay_4_yr,Delay_6_yr $\}=\{.8,2,0\}=$,

0 , //CANFuelDelay.None

$2, \quad$ // CANFuelDelay.Delay 2 yr

4, // CANFuelDelay.Delay_4_yr

6; $\quad$ // CANFuelDelay.Delay_6_yr

//Other MOX fuel plant delay

chance OthFuelDelay. $\{$ None,Delay_2_yr,Delay_4_yr,Delay_6_yr $\}=\{.5,3,1\}=$,
0 ,
// OthFuelDelay.None
2 ,
// OthFuelDelay.Delay_2_yr
4 ,
// OthFuelDelay.Delay_4_yr
6
// OthFuelDelay.Delay_6_yr

//Delay adjustment (assumes both fuel fabrication facility and

// reactor are on critical path, delay $=\max$ (fuel plant delay, reactor delay)

value GEDelayAdjust=max(OthFuelDelay-(GELicDelay+GEConstDelay+LicFailTime),0);

value WDelayAdjust=max(OthFuelDelay-(WLicDelay+WConstDelay+LicFailTime),0);

value CEDelayAdjust=max(OthFuelDelay-(CELicDelay+CEConstDelay+LicFailTime),0);

value GADelayAdjust=max(OthFuelDelay-(GALicDelay+GAConstDelay+LicFailTime),0);

value EXGEDelayAdjust=max(OthFuelDelay-(EXGELicDelay+EXGEConstDelay+LicFailTime),0); 
value EXWDelayAdjust=max(OthFuelDelay-(EXWLicDelay+EXWConstDelay+LicFailTime),0); value EXCEDelayAdjust=max(OthFuelDelay-(EXCELicDelay+EXCEConstDelay+LicFailTime),0); value EXCANDelayAdjust=max(CANFuelDelay-

(EXCANLicDelay+EXCANConstDelay+LicFailTime),0);

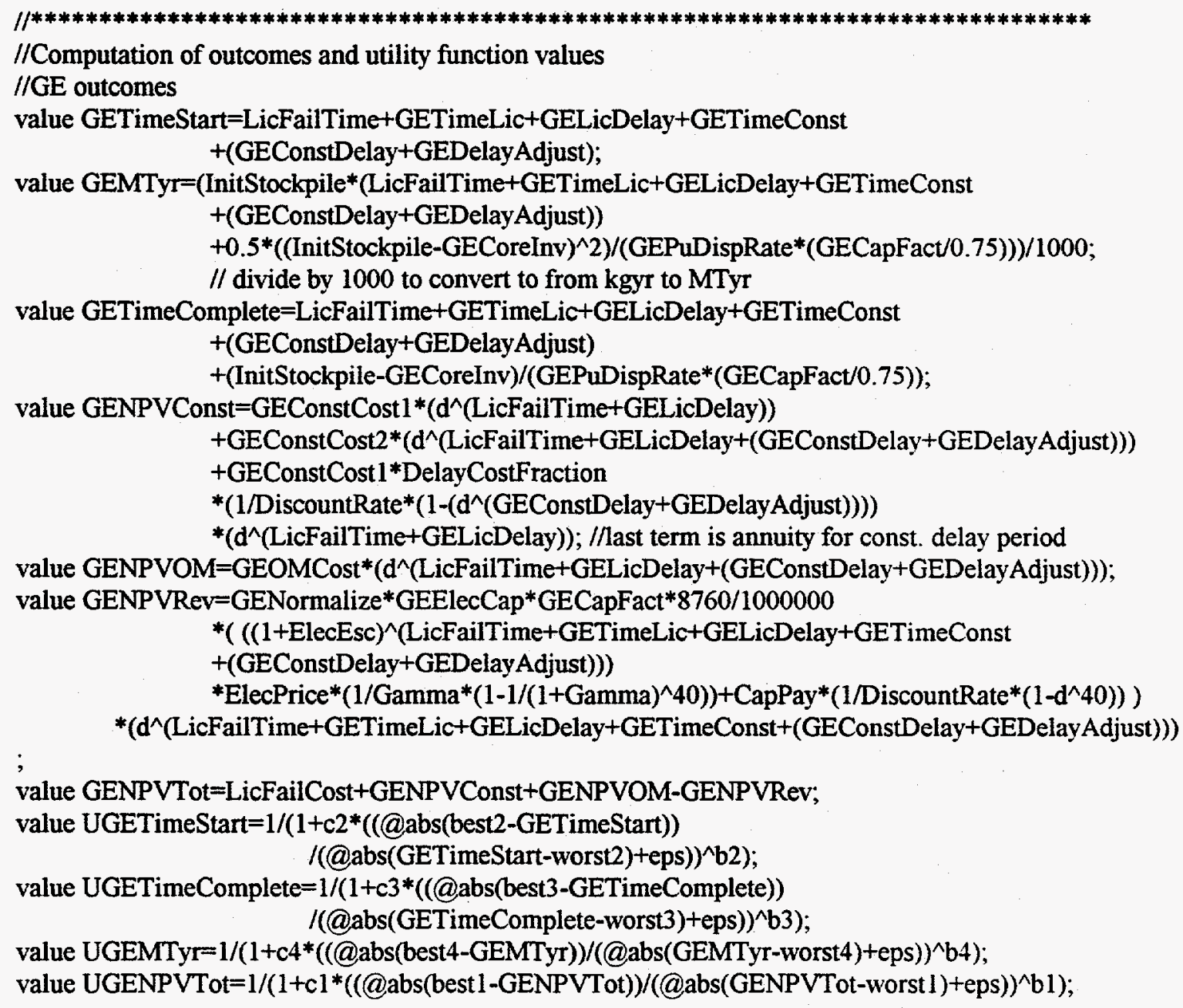


*(d^(LicFailTime+WTimeLic+WLicDelay+WTimeConst+(WConstDelay+WDelayAdjust))); value WNPVTot=LicFailCost+WNPVConst+WNPVOM-WNPVRev; value UWTimeStart=1/(1+c2*((@abs(best2-WTimeStart)) /(@abs(WTimeStart-worst2)+eps))^b2);

value UWTimeComplete=1/(1+c3*((@abs(best3-WTimeComplete) $)$ $\left./(@ a b s(W T i m e C o m p l e t e-w o r s t 3)+e p s))^{\wedge} b 3\right)$;

value UWMTyr=1/(1+c4*((@abs(best4-WMTyr))/(@abs(WMTyr-worst4)+eps))^b4);

value UWNPVTot=1/(1+c1*((@abs(best1-WNPVTot))/(@abs(WNPVTot-worst1)+eps))^b1);

//CE outcomes

value CETimeStart $=$ LicFailTime + CETimeLic + CELicDelay + CETimeConst + (CEConstDelay+CEDelayAdjust);

value CEMTyr $=($ InitStockpile* $($ LicFailTime+CETimeLic + CELicDelay+CETimeConst $+($ CEConstDelay+CEDelayAdjust)) $+0.5^{*}(($ InitStockpile-CECoreInv)^2)/(CEPuDispRate*(CECapFact/0.75)) $) / 1000$;

value CETimeComplete=LicFailTime + CETimeLic + CELicDelay+CETimeConst + (CEConstDelay+CEDelayAdjust) +(InitStockpile-CECoreInv)/(CEPuDispRate*(CECapFact/0.75));

value $\mathrm{CENPVConst}=\mathrm{CEConstCost}{ }^{*}\left(\mathrm{~d}^{\wedge}(\right.$ LicFailTime $+\mathrm{CEL}$ icDelay $\left.)\right)$ + CEConstCost $2 *\left(d^{\wedge}(\right.$ LicFailTime+CELicDelay+(CEConstDelay+CEDelayAdjust $\left.\left.)\right)\right)$

+ CEConstCost $1 *$ DelayCostFraction*(1/DiscountRate

$\left.*\left(1-\left(d^{\wedge}(C E C o n s t D e l a y+C E D e l a y A d j u s t)\right)\right)\right)^{*}\left(d^{\wedge}(\right.$ LicFailTime $\left.+C E L i c D e l a y)\right)$;

value CENPVOM $=$ CEOMCost $*\left(\mathrm{~d}^{\wedge}(\right.$ LicFailTime+CELicDelay+(CEConstDelay+CEDelayAdjust $\left.)\right)$; value $\mathrm{CENPVRev}=$ CENormalize* ${ }^{*}$ EElecCap*CECapFact*8760/1000000

${ }^{*}\left(\left((1+\text { ElecEsc })^{\wedge}\right.\right.$ (LicFailTime+CETimeLic+CELicDelay+CETimeConst $+($ CEConstDelay+CEDelayAdjust) $))$

*ElecPrice*(1/Gamma*(1-1/(1+Gamma)^40))+CapPay*(1/DiscountRate*(1-d^40)) )

*(d^(LicFailTime+CETimeLic+CELicDelay+CETimeConst+(CEConstDelay+CEDelayAdjust))); value CENPVTot $=$ LicFailCost + CENPVConst+CENPVOM-CENPVRev; value UCETimeStart=1/(1+c2*((@abs(best2-CETimeStart)) /(@abs(CETimeStart-worst2)+eps))^b2);

value UCETimeComplete=1/(1+c3*((@abs(best3-CETimeComplete) $)$ ((@abs(CETimeComplete-worst3)+eps) ) ${ }^{\circ} 3$ );

value UCEMTyr=1/(1+c4*((@abs(best4-CEMTyr))/(@abs(CEMTyr-worst4)+eps))^b4); value UCENPVTot=1/(1+c1*((@abs(best1-CENPVTot) $\left.) /(@ a b s(C E N P V T o t-w o r s t 1)+e p s))^{\wedge} b 1\right)$;

//GA outcomes

value GATimeStart=LicFailTime+GATimeLic+GALicDelay+GATimeConst +(GAConstDelay+GADelayAdjust);

value GAMTyr=(InitStockpile*(LicFailTime+GATimeLic+GALicDelay+GATimeConst $+($ GAConstDelay+GADelayAdjust)) $+0.5^{*}(($ InitStockpile-GACoreInv)^2)/(GAPuDispRate*(GACapFact/0.75)))/1000;

value GATimeComplete $=$ LicFailTime+GATimeLic+GALicDelay+GATimeConst + (GAConstDelay+GADelayAdjust) +(InitStockpile-GACoreInv)/(GAPuDispRate*(GACapFact/0.75));

value GANPVConst $=$ GAConstCost ${ }^{*}\left(\mathrm{~d}^{\wedge}(\right.$ LicFailTime+GALicDelay $\left.)\right)$ +GAConstCost $2 *\left(d^{\wedge}(\right.$ LicFailTime+GALicDelay+(GAConstDelay+GADelayAdjust) $\left.)\right)$ +GAConstCost $1 *$ DelayCostFraction* $(1 /$ DiscountRate *(1-(d^(GAConstDelay+GADelayAdjust $))))^{*}\left(\mathrm{~d}^{\wedge}(\right.$ LicFailTime + GALicDelay $\left.)\right)$; value GANPVOM=GAOMCost* ${ }^{*}\left(\mathrm{~d}^{\wedge}(\right.$ LicFailTime+GALicDelay+(GAConstDelay+GADelayAdjust $\left.\left.)\right)\right)$; value GANPVRev $=$ GANormalize* GAElecCap*GACapFact*8760/1000000

*( $\left((1+\text { ElecEsc })^{\wedge}(\right.$ LicFailTime+GATimeLic+GALicDelay+GATimeConst $+($ GAConstDelay+GADelayAdjust) $))$

*ElecPrice* $\left(1 /\right.$ Gamma*$\left.^{*}\left(1-1 /(1+\text { Gamma })^{\wedge} 40\right)\right)+$ CapPay*$^{*}\left(1 /\right.$ DiscountRate* $\left.\left.^{*}\left(1-\mathrm{d}^{\wedge} 40\right)\right)\right)$ 
*(d^(LicFailTime+GATimeLic+GALicDelay+GATimeConst+(GAConstDelay+GADelayAdjust)

));

value GANPVTot=LicFailCost+GANPVConst+GANPVOM-GANPVRev;

value UGATimeStart=1/(1+c2*((@abs(best2-GATimeStart))

$/(@$ abs(GATimeStart-worst2)+eps))^b2);

value UGATimeComplete=1/(1+c3*((@abs(best3-GATimeComplete) $)$

$/(@ \text { abs(GATimeComplete-worst3)+eps) })^{\wedge}$ b3);

value UGAMTyr=1/(1+c4*((@abs(best4-GAMTyr))/(@abs(GAMTyr-worst4)+eps))^b4);

value UGANPVTot=1/(1+c1*((@abs(best1-GANPVTot))/(@abs(GANPVTot-worst1)+eps))^bl);

//EXGE outcomes

value EXGETimeStart=LicFailTime+EXGETimeLic+EXGELicDelay+EXGETimeConst

+ (EXGEConstDelay+EXGEDelayAdjust);

value EXGEMTyr $=($ InitStockpile*(LicFailTime+EXGETimeLic+EXGELicDelay+EXGETimeConst

$+($ EXGEConstDelay+EXGEDelayAdjust))

$+0.5 *$ (InitStockpile-

EXGECoreInv) ${ }^{\wedge}$ )/(EXGEPuDispRate*(EXGECapFact/0.75)))/1000;

value EXGETimeComplete=LicFailTime+EXGETimeLic+EXGELicDelay+EXGETimeConst

+ (EXGEConstDelay+EXGEDelayAdjust)

+(InitStockpile-EXGECoreInv)/(EXGEPuDispRate*(EXGECapFact/0.75));

value EXGENPVConst $=$ EXGEConstCost ${ }^{*}\left(\mathrm{~d}^{\wedge}\left(\mathrm{LicF}^{*}\right.\right.$ ailTime + EXGELicDelay $\left.)\right)$

+EXGEConstCost $2 *\left(d^{\wedge}(\right.$ LicFailTime+EXGELicDelay+(EXGEConstDelay+EXGEDelayAdjust))

)

+EXGEConstCost $1 *$ DelayCostFraction*(1/DiscountRate

*(1-(d^(EXGEConstDelay+EXGEDelayAdjust $))))^{*}\left(d^{\wedge}(\right.$ LicFailTime+EXGELicDelay $\left.)\right)$;

value EXGENPVOM=EXGEOMCost

*(d^(LicFailTime+EXGELicDelay+(EXGEConstDelay+EXGEDelayAdjust)));

value EXGENPVRev=EXGENormalize*EXGEElecCap*EXGECapFact*8760/1000000

* $\left(\left((1+\text { ElecEsc })^{\wedge}(\right.\right.$ LicFailTime+EXGETimeLic+EXGELicDelay+EXGETimeConst

$+($ EXGEConstDelay+EXGEDelayAdjust)))

*ElecPrice* $\left(1 /\right.$ Gamma*$^{*}\left(1-1 /\left(1+\right.\right.$ Gamma $\left.\left.^{\wedge} 40\right)\right)+$ CapPay*$^{*}\left(1 /\right.$ DiscountRate $\left.\left.^{*}\left(1-d^{\wedge} 40\right)\right)\right)$

* $\left(\mathrm{d}^{\wedge}(\right.$ LicFailTime+EXGETimeLic+EXGELicDelay+EXGETimeConst

$+($ EXGEConstDelay+EXGEDelayAdjust)));

value EXGENPVTot=LicFailCost+EXGENPVConst+EXGENPVOM-EXGENPVRev;

value UEXGETimeStart=1/(1+c2*((@abs(best2-EXGETimeStart))

/(@abs(EXGETimeStart-worst2)+eps)) $)^{\wedge}$ 2);

value UEXGETimeComplete=1/(1+c3*((@abs(best3-EXGETimeComplete))

$/\left((\text { abs(EXGETimeComplete-worst3)+eps) })^{\wedge} \mathrm{b} 3\right)$;

value UEXGEMTyr=1/(1+c4*((@abs(best4-EXGEMTyr))/(@abs(EXGEMTyr-worst4)+eps))^b4);

value UEXGENPVTot=1/(1+c1*((@abs(bestl-EXGENPVTot))/(@abs(EXGENPVTot-worst1)+eps))^bl);

//EXW outcomes

value EXWTimeStart=LicFailTime+EXWTimeLic+EXWLicDelay+EXWTimeConst

+ (EXWConstDelay+EXWDelayAdjust);

value EXWMTyr=(InitStockpile*(LicFailTime+EXWTimeLic+EXWLicDelay+EXWTimeConst

$+($ EXWConstDelay+EXWDelayAdjust))

$+0.5^{*}(($ InitStockpile-EXWCoreInv)^2)/(EXWPuDispRate*(EXWCapFact/0.75)))/1000;

value EXWTimeComplete=LicFailTime+EXWTimeLic+EXWLicDelay+EXWTimeConst

+ (EXWConstDelay+EXWDelayAdjust)

+(InitStockpile-EXWCoreInv)/(EXWPuDispRate*(EXWCapFact/0.75));

value EXWNPVConst $=$ EXWConstCost $1^{*}\left(\mathrm{~d}^{\wedge}(\right.$ LicFailTime + EXWLicDelay))

+ EXWConstCost $2 *\left(\mathrm{~d}^{\wedge}(\right.$ LicFailTime+EXWLicDelay+(EXWConstDelay+EXWDelayAdjust $\left.\left.)\right)\right)$

+ EXWConstCost ${ }^{*}$ DelayCostFraction* $(1 /$ DiscountRate

$*\left(1-\left(d^{\wedge}(\right.\right.$ EXWConstDelay+EXWDelayAdjust $\left.\left.\left.)\right)\right)\right)^{*}\left(d^{\wedge}(\right.$ LicFailTime+EXWLicDelay $\left.)\right) ;$ 
value EXWNPVOM=EXWOMCost ${ }^{*}\left(\mathrm{~d}^{\wedge}(\right.$ LicFailTime+EXWLicDelay $+($ EXWConstDelay+EXWDelayAdjust)));

value EXWNPVRev $=$ EXWNormalize*EXWElecCap*EXWCapFact*8760/1000000

${ }^{*}\left(\left((1+\text { ElecEsc })^{\wedge}\right.\right.$ (LicFailTime+EXWTimeLic+EXWLicDelay+EXWTimeConst $+($ EXWConstDelay+EXWDelayAdjust)) $)$

*ElecPrice*(1/Gamma*(1-1/(1+Gamma)^40))+CapPay*(1/DiscountRate*(1-d^40)) )

*(d^(LicFailTime+EXWTimeLic+EXWLicDelay+EXWTimeConst

$+($ EXWConstDelay+EXWDelayAdjust)));

value EXWNPVTot $=$ LicFailCost+EXWNPVConst+EXWNPVOM-EXWNPVRev;

value UEXWTimeStart=1/(1+c2*((@abs(best2-EXWTimeStart)) /(@abs(EXWTimeStart-worst2)+eps))^b2);

value UEXWTimeComplete=1/(1+c3*((@abs(best3-EXWTimeComplete)) /(@abs(EXWTimeComplete-worst3)+eps))^b3);

value UEXWMTyr=1/(1+c4*((@abs(best4-EXWMTyr))/(@abs(EXWMTyr-worst4)+eps))^b4); value UEXWNPVTot=1/(1+c1*((@abs(bestl-EXWNPVTot))/(@abs(EXWNPVTot-worst1)+eps))^b1);

//EXCE outcomes

value EXCETimeStart=LicFailTime+EXCETimeLic+EXCELicDelay+EXCETimeConst +(EXCEConstDelay+EXCEDelayAdjust);

value EXCEMTyr $=$ (InitStockpile*(LicFailTime+EXCETimeLic+EXCELicDelay+EXCETimeConst $+($ EXCEConstDelay+EXCEDelayAdjust)) $+0.5 *$ ((InitStockpile-

EXCECoreInv)^2)/(EXCEPuDispRate*(EXCECapFact/0.75)))/1000;

value EXCETimeComplete=LicFailTime+EXCETimeLic+EXCELicDelay+EXCETimeConst + (EXCEConstDelay+EXCEDelayAdjust) +(InitStockpile-EXCECoreInv)/(EXCEPuDispRate*(EXCECapFact/0.75));

value EXCENPVConst $=$ EXCEConstCost $1^{*}\left(\mathrm{~d}^{\wedge}(\mathrm{LicF}\right.$ ailTime+EXCELicDelay $\left.)\right)$

+ EXCEConstCost $2 *\left(d^{\wedge}(\right.$ LicFailTime+EXCELicDelay+(EXCEConstDelay+EXCEDelayAdjust)) + EXCEConstCost $1 *$ DelayCostFraction* $(1 /$ DiscountRate

*(1-(d^(EXCEConstDelay+EXCEDelayAdjust $))){ }^{*}\left(d^{\wedge}(\right.$ LicFailTime+EXCELicDelay $\left.)\right) ;$ value EXCENPVOM=EXCEOMCost *(d^(LicFailTime+EXCELicDelay+(EXCEConstDelay+EXCEDelayAdjust)));

value EXCENPVRev=EXCENormalize*EXCEElecCap*EXCECapFact*8760/1000000

*( ( (1+ElecEsc)^(LicFailTime+EXCETimeLic+EXCELicDelay+EXCETimeConst $+($ EXCEConstDelay+EXCEDelayAdjust $)))$

*ElecPrice*(1/Gamma*(1-1/(1+Gamma)^40))+CapPay*(1/DiscountRate*(1-d^40)) )

*( $\mathrm{d}^{\wedge}($ LicFailTime+EXCETimeLic+EXCELicDelay+EXCETimeConst $+($ EXCEConstDelay+EXCEDelayAdjust)));

value EXCENPVTot=LicFailCost+EXCENPVConst+EXCENPVOM-EXCENPVRev;

value UEXCETimeStart=1/(1+c2*((@abs(best2-EXCETimeStart)) /(@abs(EXCETimeStart-worst2)+eps) $)^{\wedge}$ b2); value UEXCETimeComplete=1/(1+c3*((@abs(best3-EXCETimeComplete)) /(@abs(EXCETimeComplete-worst3)+eps))^b3);

value UEXCEMTyr=1/(1+c4*((@abs(best4-EXCEMTyr))/(@abs(EXCEMTyr-worst4)+eps) $)^{\wedge}$ b4); value UEXCENPVTot=1/(1+cl*((@abs(best l-EXCENPVTot))/(@abs(EXCENPVTot-worstl)+eps))^bl);

//EXCAN outcomes

value EXCANTimeStart=LicFailTime+EXCANTimeLic+EXCANLicDelay+EXCANTimeConst $+($ EXCANConstDelay+EXCANDelayAdjust);

value

EXCANMTyr $=($ InitStockpile* $($ LicFailTime+EXCANTimeLic+EXCANLicDelay+EXCANTimeConst $+($ EXCANConstDelay+EXCANDelayAdjust)) $+0.5 *(($ InitStockpile-

EXCANCoreInv $\left.)^{\wedge} 2\right) /($ EXCANPuDispRate*(EXCANCapFact/0.80)))/1000; 
value EXCANTimeComplete=LicFailTime+EXCANTimeLic+EXCANLicDelay+EXCANTimeConst + (EXCANConstDelay+EXCANDelayAdjust) +(InitStockpile-EXCANCoreInv)/(EXCANPuDispRate*(EXCANCapFact/0.80)); $/ / 80 \%$ capacity factor

value EXCANNPVConst $=$ EXCANConstCost $l^{*}\left(\mathrm{~d}^{\wedge}(\right.$ LicFailTime+EXCANLicDelay $\left.)\right)$

+ EXCANConstCost $2 *\left(d^{\wedge}(\right.$ LicFailTime+EXCANLicDelay

$+($ EXCANConstDelay+EXCANDelayAdjust)))

+EXCANConstCost $1 *$ DelayCostFraction*(1/DiscountRate

*(1-(d^(EXCANConstDelay+EXCANDelayAdjust $))))$

*(d^(LicFailTime+EXCANLicDelay));

value EXCANNPVOM=EXCANOMCost* $\left(d^{\wedge}(\right.$ LicFailTime+EXCANLicDelay

+(EXCANConstDelay+EXCANDelayAdjust)));

value EXCANNPVRev=EXCANNormalize*EXCANElecCap*EXCANCapFact*8760/1000000

* $\left(\left((1+\text { ElecEsc })^{\wedge}(\right.\right.$ LicFailTime+EXCANTimeLic+EXCANLicDelay+EXCANTimeConst $+($ EXCANConstDelay+EXCANDelayAdjust $)))$

*ElecPrice*(1/Gamma*(1-1/(1+Gamma)^40))+CapPay*(1/DiscountRate*(1-d^40)) )

$*\left(d^{\wedge}(\right.$ LicFailTime+EXCANTimeLic+EXCANLicDelay+EXCANTimeConst

$+($ EXCANConstDelay+EXCANDelayAdjust)));

value EXCANNPVTot=LicFailCost+EXCANNPVConst+EXCANNPVOM-EXCANNPVRev;

value UEXCANTimeStart=1/(1+c2* $((@$ abs(best2-EXCANTimeStart)) $\left./(@ a b s(E X C A N T i m e S t a r t-w o r s t 2)+e p s))^{\wedge} b 2\right)$;

value UEXCANTimeComplete=1/(1+c3*((@abs(best3-EXCANTimeComplete))

/(@abs(EXCANTimeComplete-worst3)+eps) ) $)^{\mathrm{b}} 3$ );

value UEXCANMTyr=1/(1+c4*((@abs(best4-EXCANMTyr) $\left.) /(@ a b s(E X C A N M T y r-w o r s t 4)+e p s))^{\wedge} b 4\right)$;

value UEXCANNPVTot=1/(1+cl*((@abs(best1-EXCANNPVTot))/(@abs(EXCANNPVTot-

worst1)+eps))^b1);

//decision nodes for failure of first reactor licensing attempt decision GEFailLic. $\{$ W,CE,GA,EXGE,EXW,EXCE,EXCAN\}; decision WFailLic. \{GE,CE,GA,EXGE,EXW,EXCE,EXCAN\}; decision CEFailLic. \{GE,W,GA,EXGE,EXW,EXCE,EXCAN\}; decision GAFailLic. $\{\mathrm{GE}, \mathrm{W}, \mathrm{CE}, \mathrm{EXGE}, \mathrm{EXW}, \mathrm{EXCE}, \mathrm{EXCAN}\}$; decision EXGEFaillic. $\{$ GE,W,CE,GA,EXW,EXCE,EXCAN\}; decision EXWFailLic. \{GE,W,CE,GA,EXGE,EXCE,EXCAN\}; decision EXCEFailLic. \{GE,W,CE,GA,EXGE,EXW,EXCAN\}; decision EXCANFailLic. $\{$ GE,W,CE,GA,EXGE,EXW,EXCE\};

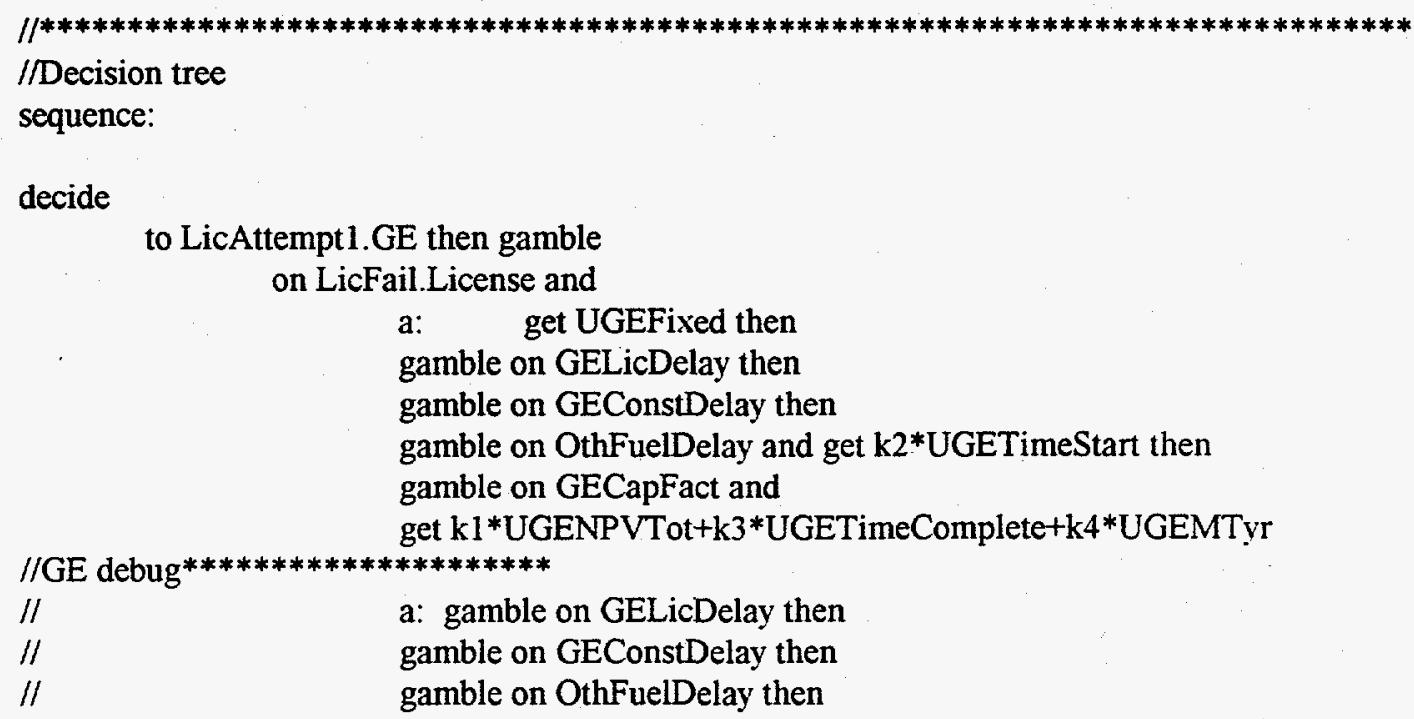


on LicFail.NoLicense then decide

to GEFaillic.W and

b: $\quad$ get UWFixed then

gamble on WLicDelay then gamble on WConstDelay then

gamble on OthFuelDelay and get k2*UWTimeStart then gamble on WCapFact and

//W debug********************* get k1*UWNPVTot+k3*UWTimeComplete+k4*UWMTyr

/I

/I

/I

/I

$/ / * * * * * * * * * * * * * * * * * * * * * * * * * * * *$

to GEFaillic.CE and

c: get UCEFixed then

gamble on CELicDelay then gamble on CEConstDelay then gamble on OthFuelDelay and get k2*UCETimeStart then gamble on CECapFact and

$/ / \mathrm{CE}$ debug****************

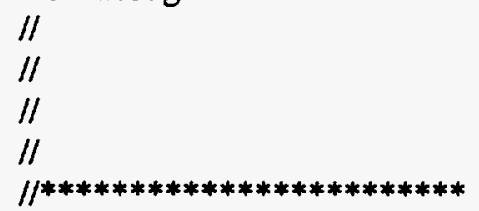

get $\mathrm{kl}$ *UCENPVTot+k3*UCETimeComplete+k4*UCEMTyr

c: gamble on CELicDelay then gamble on CEConstDelay then gamble on OthFuelDelay then gamble on CECapFact and get CENPVTot

to GEFailLic.GA and

d: get UGAFixed then

gamble on GALicDelay then gamble on GAConstDelay then gamble on OthFuelDelay and get $\mathrm{k} 2 *$ UGATimeStart then gamble on GACapFact and $/ / \mathrm{GA}$ debug********************* get k1*UGANPVTot+k3*UGATimeComplete+k4*UGAMTyr
II
II
II
11
$/ / * * * * * * * * * * * * * * * * * * * * * * * * * * * * *$
d: gamble on GALicDelay then gamble on GAConstDelay then gamble on OthFuelDelay then gamble on GACapFact and get GANPVTot

to GEFailLic.EXGE and

e: get UEXGEFixed then gamble on EXGELicDelay then gamble on EXGEConstDelay then gamble on OthFuelDelay and get $\mathbf{k} 2 * \mathrm{UEXGETimeStart} \mathrm{then}$ gamble on EXGECapFact and get

$\mathrm{k} 1 *$ UEXGENPVTot+k3*UEXGETimeComplete+k4*UEXGEMTyr $/ / \mathrm{EXGE}$ debug*********************

II

$1 /$

/I e: gamble on EXGELicDelay then gamble on EXGEConstDelay then gamble on OthFuelDelay then 


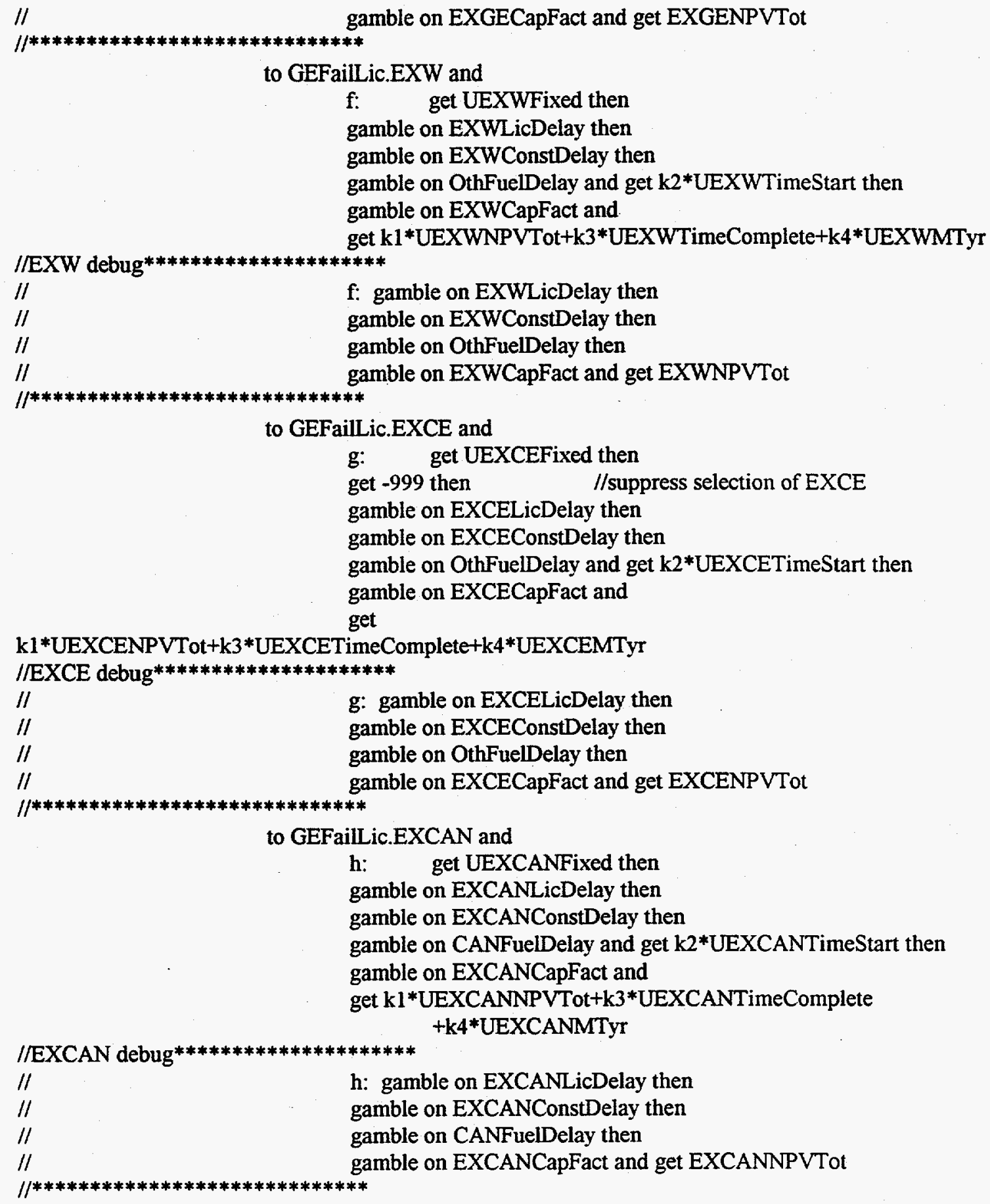

to LicAttempt 1 .W then gamble on LicFail.License then perform $b$

on LicFail.NoLicense then decide

to WFailLic.GE and perform a

to WFailLic.CE and perform $c$

to WFailLic.GA and perform d 
to WFaillic.EXGE and perform e

to WFailLic.EXW and

perform $f$

to WFailLic.EXCE and perform $g$

to WFaillic.EXCAN and perform $h$

to LicAttempt1.CE then gamble

on LicFail.License then

perform c

on LicFail.NoLicense then decide

to CEFaillic.GE and

perform a

to CEFaillic.W and

perform $b$

to CEFaillic.GA and

perform d

to CEFailLic.EXGE and perform e

to CEFailLic.EXW and perform $f$

to CEFailLic.EXCE and perform $\mathbf{g}$

to CEFailLic.EXCAN and perform $h$

to LicAttempt1.GA then gamble

on LicFail.License then

perform d

on LicFail.NoLicense then decide

to GAFaillic.GE and perform a

to GAFaillic.W and perform $b$

to GAFailLic.CE and perform $c$

to GAFailLic.EXGE and perform e

to GAFailLic.EXW and perform $f$

to GAFailLic.EXCE and perform $g$

to GAFailLic.EXCAN and perform $\mathrm{h}$

to LicAttempt1.EXGE then gamble

on LicFail.License then

perform e

on LicFail.NoLicense then decide

to EXGEFailLic.GE and perform a

to EXGEFailLic.W and perform $b$

to EXGEFailLic.CE and perform $\mathrm{c}$ 
to

EXGEFailLic.GA and perform d

to EXGEFaillic.EXW and perform $f$

to EXGEFaillic.EXCE and perform $\mathrm{g}$

to EXGEFailLic.EXCAN and perform $h$

to LicAttemptl.EXW then gamble

on LicFail.License then

perform $f$

on LicFail.NoLicense then decide

to

EXWFaillic.GE and perform a

to EXWFailLic.W and perform b

to EXWFaillic.CE and perform c

to EXWFailLic.GA and perform d

to EXWFailLic.EXGE and perform e

to EXWFailLic.EXCE and perform $\mathbf{g}$

to EXWFailLic.EXCAN and perform $h$

to LicAttempt1.EXCE then gamble

on LicFail.License then perform $g$

on LicFail.NoLicense then decide

to EXCEFailLic.GE and perform a

to EXCEFaillic.W and perform b

to EXCEFaillic.CE and perform c

to EXCEFaillic.GA and perform d

to EXCEFaillic.EXGE and perform e

to EXCEFailLic.EXW and perform $f$

to EXCEFaillic.EXCAN and perform $h$

to LicAttempt1.EXCAN then gamble on LicFail.License then perform $h$ on LicFail.NoLicense then decide EXCANFailLic.GE and perform a

to EXCANFaillic.W and perform $b$

to EXCANFailLic.CE and perform $\mathrm{c}$ 
EXCANFaillic.GA and perform d

to EXCANFailLic.EXGE and perform e

to EXCANFailLic.EXW and perform $f$

to EXCANFailLic.EXCE and perform $g$ 